\title{
\begin{tabular}{l|l} 
Mitraries & DSpace@MIT
\end{tabular}
}

\author{
MIT Open Access Articles
}

Performance of the CMS hadron calorimeter with cosmic ray muons and LHC beam data

The MIT Faculty has made this article openly available. Please share how this access benefits you. Your story matters.

Citation: Chatrchyan, S. et al. "Performance of the CMS hadron calorimeter with cosmic ray muons and LHC beam data." Journal of Instrumentation 5 (March 2010): T03012 (c) 2010 IOP Publishing Ltd and SISSA

As Published: http://dx.doi.org/10.1088/1748-0221/5/03/T03012

Publisher: IOP Publishing

Persistent URL: https://hdl.handle.net/1721.1/121503

Version: Original manuscript: author's manuscript prior to formal peer review

Terms of use: Creative Commons Attribution-Noncommercial-Share Alike 


\title{
CMS Paper
}

$2010 / 01 / 01$

\section{Performance of the CMS Hadron Calorimeter with Cosmic Ray Muons and LHC Beam Data}

\author{
The CMS Collaboration»
}

\begin{abstract}
The CMS Hadron Calorimeter in the barrel, endcap and forward regions is fully commissioned. Cosmic ray data were taken with and without magnetic field at the surface hall and after installation in the experimental hall, hundred meters underground. Various measurements were also performed during the few days of beam in the LHC in September 2008. Calibration parameters were extracted, and the energy response of the HCAL determined from test beam data has been checked.
\end{abstract}





\section{Introduction}

The primary goal of the Compact Muon Solenoid (CMS) experiment [1] is to explore particle physics at the $\mathrm{TeV}$ energy scale exploiting the proton-proton collisions delivered by the Large Hadron Collider (LHC) [2]. This paper describes the performance of the CMS Hadron Calorimeter (HCAL). The measurement of the detector response started with the characterization and calibration of representative samples of all detector components in the laboratory using a test beam [3-6]. After assembly of the detector, the calibration was improved and validated using cosmic ray muons and LHC beam. The cosmic ray muon data also allowed the testing of the whole data acquisition process, up to the event reconstruction and analysis. The validation includes comparisons with Monte Carlo simulation studies, which are fundamental in understanding the detailed performance of the detector.

CMS has taken cosmic ray muon data with and without magnetic field at the surface hall in 2006, during the Magnet Test and Cosmic Challenge (MTCC) described in Ref. [7]. Recently, data were taken in the underground cavern at $\sim 100 \mathrm{~m}$ depth with the magnet off in 2007 (CRUZET: Cosmic RUn at ZEro Tesla) and with the magnet on in 2008 (CRAFT: Cosmic Run At Four Tesla [8]).

In September 2008, the LHC delivered a single circulating beam of $450 \mathrm{GeV} / c$ protons for a few days. In addition, as part of the commissioning of the LHC, the beam was also sent onto collimator targets creating accelerator produced muons. These "beam splash" events proved very useful to determine the relative timing of various the HCAL systems [9], as well as to validate the performance of several components.

Knowledge of the HCAL performance acquired during CRAFT and with beam splash data will prove crucial to the understanding of data in the early stages of LHC operation. This will allow the timely analysis of benchmark QCD processes and of known missing energy signals. Any search for new phenomena must be grounded on these benchmark analyses.

The paper is structured as follows. Section 2 provides a brief description of the Hadron Calorimeter. Section 3 discusses the impact of the magnetic field on the various parts of the HCAL subsystems. The characteristics of the basic behavior of all the HCAL subsystems and the optimization of key hardware settings are summarized in Section 4. Finally, Section 5 discusses the calibration improvements obtained with the various data samples.

\section{CMS Hadron Calorimeter}

The main CMS subsystems, in increasing radial distance from the beam intersection point (IP), are the silicon pixel and silicon strip tracker, the electromagnetic calorimeter, the hadron calorimeter, and finally the muon system. In addition, in the forward direction there are two specialized calorimeters [1] that did not participate in the cosmic ray runs and are not discussed in this paper. The detector is symmetric for positive and negative $z$ coordinates, the $z$ axis being along the beam direction with $z=0$ at the IP. The polar angle, $\theta$, is measured from the $z$ direction, and the azimuthal angle, $\phi$, is measured in the transverse plane. The pseudorapidity is $\eta \equiv-\ln \tan (\theta / 2)$.

The HCAL includes four distinct subsystems: the barrel $(\mathrm{HB})$, endcap $(\mathrm{HE})$, outer $(\mathrm{HO})$ and forward (HF) calorimeters (see Fig. 1). HB and HE, which are inside the cryostat of the CMS superconducting solenoid that provides a $3.8 \mathrm{~T}$ magnetic field, are sampling calorimeters where the absorber material is brass and the active material is scintillator. The sampling fraction is about $7 \%$. HB and HE are separated by a gap which is located approximately at a polar angle 
of 57 degrees but is not projective to the center of CMS, in order to minimize the effect of the uninstrumented gap. HB covers the $|\eta|$ range from zero to approximately 1.4 ; the $|\eta|$ range between 1.3 and 1.4 is shared by HB and HE; HE covers $|\eta|$ from 1.3 to 3.0. HB is built of 18 wedges, each of which covers 20 degrees in $\phi$, and are divided in 5 degree sectors. HE is made of brass disks, interleaved with scintillator wedges which cover 20 degrees in $\phi$, which in turn are divided in four 5 degree sectors. Because of the space constraint within the magnet cryostat, the HB thickness is limited to 5.8 hadronic interaction lengths at $\eta=0$ and increases to 10 interaction lengths at $|\eta|=1.2$. To catch the energy leakage from $\mathrm{HB}$, layers of scintillators are placed outside the solenoid cryostat: they constitute HO. About $5 \%$ of all hadrons above $100 \mathrm{GeV}$ deposit energy in $\mathrm{HO}$. In $\phi, \mathrm{HO}$ has a 12-fold structure, with each 30 degree component being divided in six 5 degree sectors. In $\eta, \mathrm{HO}$ is composed of five "rings", which follow the structure of the magnet return yoke and of the muon chambers. Ring 0 covers the $\eta$ range between -0.35 and 0.35 , Rings \pm 1 cover the $|\eta|$ range between 0.35 and 0.87 , and Rings \pm 2 cover the $|\eta|$ range between 0.87 and 1.2. The quartz fiber and steel HF calorimeter, with fibers parallel to the beam direction, covers the forward region of $|\eta|$, between 3.0 and 5.2. HF is constructed in wedges of 20 degrees and each wedge contains two $\phi$ sectors of 10 degrees. The calorimeter tower segmentation in $\eta$ and $\phi$ of $\mathrm{HB}, \mathrm{HE}$ and $\mathrm{HO}$ subsystems is $0.087 \times 0.087$ except in HE for $|\eta|$ above 1.74, where the $\eta$ segmentation ranges from 0.09 to 0.35 and the $\phi$ segmentation is 0.175 . The HF segmentation is $0.175 \times 0.175$ except for $|\eta|$ above 4.7 , where the segmentation is $0.175 \times 0.35$.

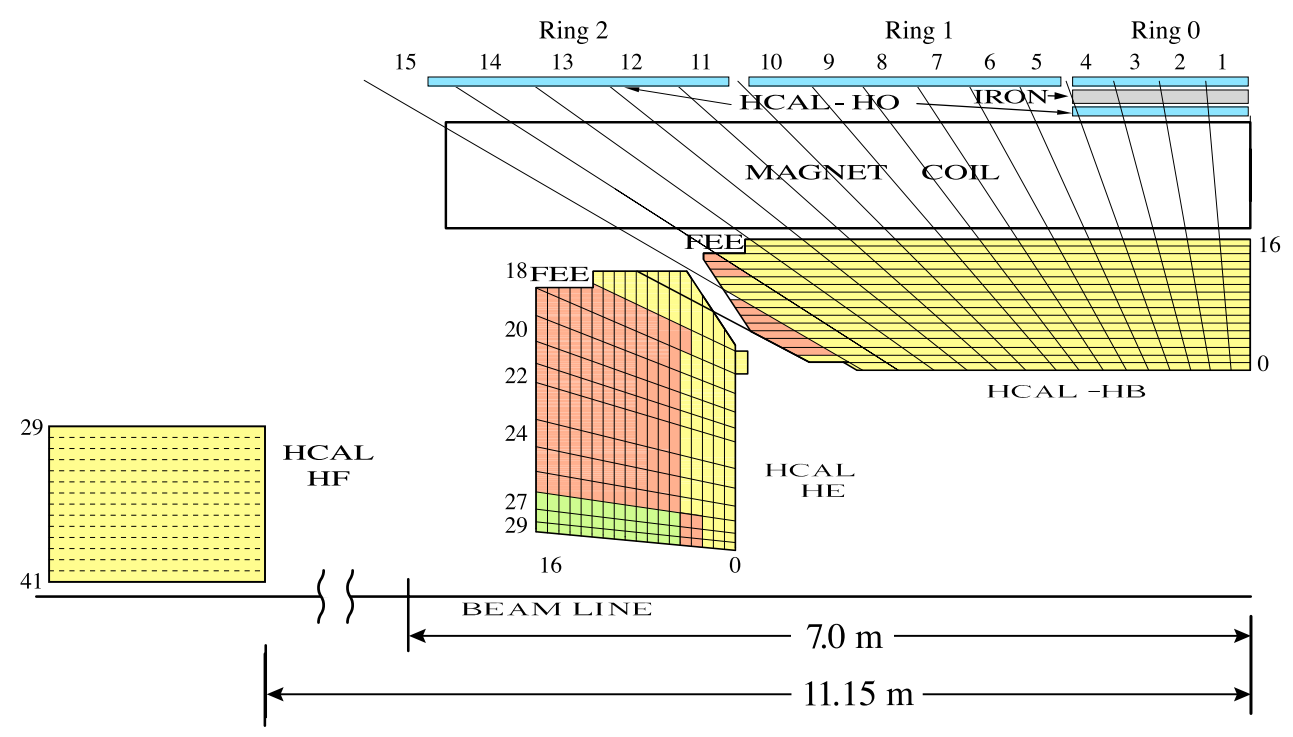

Figure 1: The CMS HCAL detector (quarter slice). "FEE" indicates the locations of the Front End Electronics for HB and HE. The signals of the tower segments with the same color are added optically, to provide the HCAL "longitudinal" segmentation. HB, HE and HF are built of 36 identical azimuthal wedges $(\Delta \phi=20$ degrees).

Figure 1 shows a schematic quarter view of the hadron calorimeter system in the barrel, endcap and forward regions. Also shown are the locations of some of the Front End Electronics (FEE). The HF FEEs (not shown) are placed around a ring at $|\eta|=3$ (tower number 29) and HO FEEs are located inside the muon detectors at various locations. Each HB and HE tower has 17 scintillator layers, except near the overlap region between HB and HE. Each scintillator tile of a tower is read out by an embedded wavelength shifting fiber and the signals are added optically. The color scheme in Fig. 1 denotes the longitudinal segmentation of the read out; all layers shown with the same color in one $\eta$ tower are summed. The optical signals for HB, HE and HO 
are detected by hybrid photodiodes (HPD) with 19 independent pixels; 18 for read out of fibers and one for monitoring. The HPDs are designed to work inside the magnetic field, provided their axes are aligned with the magnetic field. This alignment is adequate for HB and HE, but it was found that the HO HPDs were off by as much as 40 degrees. This misalignment was due to the difficulty in simulating the magnetic field inside the return yoke. Therefore, an effort is under way to study the possible replacement of the HO HPDs with silicon photomultipliers that are insensitive to magnetic fields and have a better signal to noise discrimination.

In HF, quartz fibers of two different lengths are embedded in the steel, and are read separately. The calorimeter is thus functionally subdivided into two longitudinal segments (not shown in Fig. 1). Long fibers $(165 \mathrm{~cm} \approx 10$ interaction lengths) measure the total signal coming from the full material length, whereas short fibers measure the energy deposition after $22 \mathrm{~cm}$ of steel. This allows the separation of showers generated by electrons and photons from those generated by hadrons. The photodetectors for HF are eight-stage photomultipliers (PMT) with a borosilicate glass window, which register the Cherenkov light produced by the charged shower particles in the quartz fibers.

The signals from both types of photodetectors are read by a custom-designed chip, which performs charge integration and encoding (QIE) [4]. The QIE is a non-linear multi-range analog to digital converter (ADC) that provides approximately constant fractional precision over a wide dynamic range. For each channel, the measured ADC value is converted into a charge measured in femtocoulomb (fC), using a conversion factor that was determined in the laboratory by charge injection into the QIE.

Each subsystem is equipped with laser and light emitting diodes (LED), for monitoring and calibration. In addition, the response of each HB and HE scintillator tile of each layer and each HF fiber can be measured using radioactive sources mounted on the tip of a moving wire [10].

The Electromagnetic Calorimeter (ECAL), made of lead tungstate crystals, is situated in front of $\mathrm{HB}$ and HE. The response and resolution of the CMS calorimeter system depends on both the ECAL and the HCAL, as most particles start showering in the ECAL. The hadronic to electromagnetic $(\pi / e)$ response ratios of the ECAL and the HCAL are different: typical values of $\pi / e$ for the ECAL and the HCAL are 0.61 and 0.80 at $10 \mathrm{GeV} / c$, and 0.75 and 0.85 at $100 \mathrm{GeV} / c$, respectively [6]. The ECAL and the HCAL fraction of the energy deposited in each calorimeter varies non-linearly with energy and, as a result, the raw energy measurements require substantial corrections. Results from test beam studies are reported in [3-6].

Parts of the various HCAL subsystems were exposed to beams of electrons, pions, protons and muons, to measure their characteristics and to obtain a reference calibration. An ECAL module was also included in the test beam setup. The hadronic energy resolution of the barrel HCAL and ECAL combination being parameterized as $\sigma / E=a / \sqrt{E} \oplus b$, where $a$ corresponds to a stochastic term and $b$ to a constant term, one measures $a=0.847 \pm 0.016 \mathrm{GeV}^{\frac{1}{2}}$ and $b=$ $0.074 \pm 0.008$ [4], with $E$ measured in GeV. The energy resolution in the endcaps is similar to that in the barrel. The corresponding values for HF are $a=1.98 \mathrm{GeV}^{\frac{1}{2}}$ and $b=0.09$ [3]. Since the forward jets typically have very high energies, the stochastic term may be higher for HF than for the other calorimeters, while still providing the required energy resolution [3].

\section{Impact of the Magnetic Field on the HCAL Response}

About 270 million cosmic ray muons were recorded during CRAFT with the magnetic field on. Typically, a muon event is triggered by the muon chambers and the muon momentum is 
measured by the central tracker and muon chambers. Clean signals were measured in most of the HCAL towers. The cosmic ray muons are used to measure the HPD gain to an accuracy of about $5 \%$ for $\mathrm{HB}$ and $\mathrm{HO}$ towers, and 10\% for HE. Calibration corrections based on the study of the cosmic ray muon samples have been derived and applied for $\mathrm{HB}$ and $\mathrm{HO}$, as discussed in Section 5 ,

Brightening corrections, of about 8-10\%, applied to $\mathrm{HB}$ and $\mathrm{HE}$ calibration parameters to take into account the effects of the magnetic field were already obtained during MTCC, where it was possible to perform precise measurements using radioactive sources [1]. Cosmic ray muon data from CRUZET and CRAFT allowed to test these corrections and to study the impact of the strong magnetic field in other parts of the HCAL system.
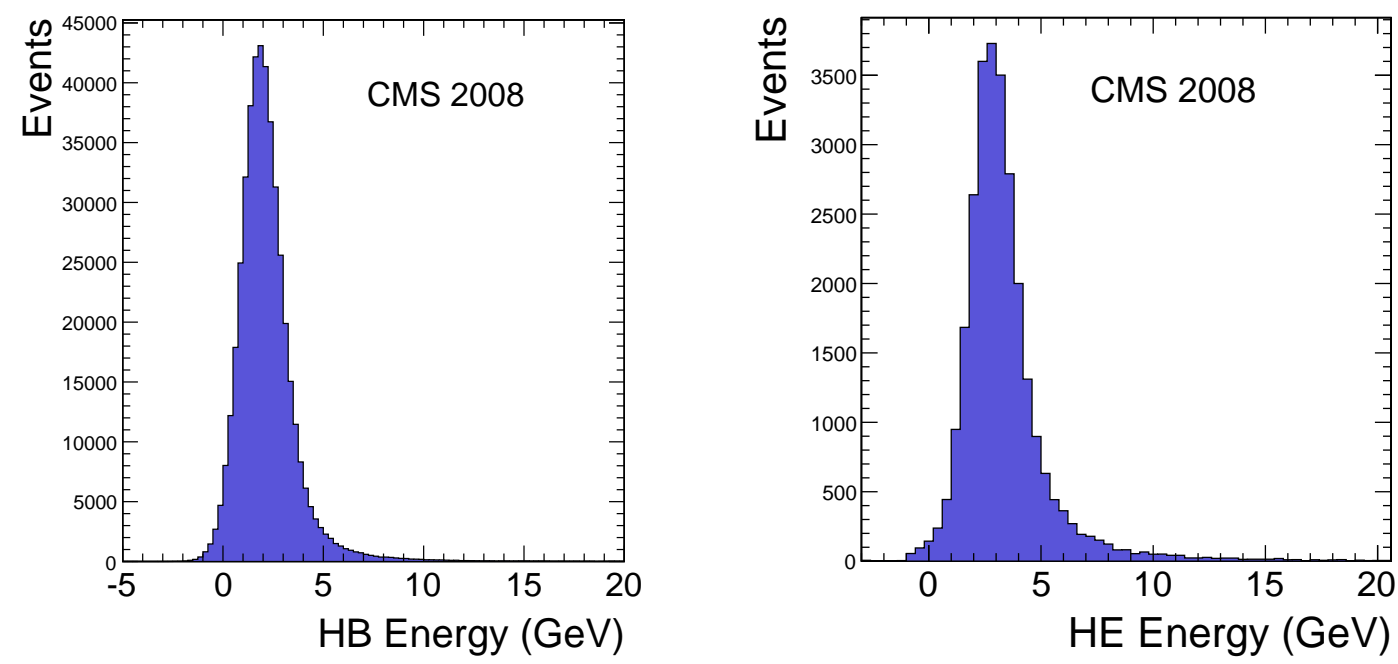

Figure 2: Cosmic ray muon energy deposition measured in $\mathrm{HB}$ (left) and HE (right) with $B=3.8 \mathrm{~T}$ for muon momenta above $7 \mathrm{GeV} / c$. HB and $\mathrm{HE}$ data samples consist of 450 and 27 thousand events, respectively.

\subsection{The Barrel and Endcap Hadron Calorimeters}

A single muon traversing the HCAL deposits a few $\mathrm{GeV}$ in $\mathrm{HB}$ and HE. Figure 2 shows the energy deposited in $\mathrm{HB}$ and HE by muons with momenta above $7 \mathrm{GeV} / c$, during CRAFT with the $3.8 \mathrm{~T}$ magnetic field. The means are consistent with the muon minimum ionizing signals measured for segments of the calorimeters in test beams [4, 6], without magnetic field, for a wide momentum range after taking into account magnetic field effects, correcting the signal for the muon path length and normalizing to the thickness of $\mathrm{HB}$ at $\eta=0$. The width of the distributions of the cosmic ray muons are broadened by the momentum spread of cosmic ray muons, since the energy deposition increases with momentum due to the relativistic rise. The measurement of the energy deposition by cosmic ray muons is performed using either the electron or the pion calibration of the calorimeter. As an example, the mean energy deposition of $150 \mathrm{GeV} / c$ muons is calculated to be $2.4 \mathrm{GeV}(2.8 \mathrm{GeV})$ if the electron (pion) calibration is used. For Fig. 2 , the pion calibration was used. The average values of the energy deposition is lower than $2.8 \mathrm{GeV}$ because the average muon momenta is well below $100 \mathrm{GeV} / c$ (see momentum spectrum given in Fig. 10). 
It is known [11-15], that the presence of a magnetic field increases the signal measured in scintillator based calorimeters. There are three sources contributing to this effect. The first of them is due to a chemical effect that causes an intrinsic brightness of the scintillator. Brightening is due to the polymer base of the scintillator and saturates at about $2 \mathrm{~T}$. The maximum signal increase due to brightness ranges between $5 \%$ and $8 \%$, depending on the composition of the plastic [15]. The field value at $\mathrm{HB}$ and $\mathrm{HE}$ is $3.8 \mathrm{~T}$, which is well above the saturation value. The second source of signal increase is the curling of knock-on electrons in the magnetic field, which results in an increased path length of the electrons, and enhances the signal by $1-2 \%$. Therefore, the signal increase depends on the nature of the particle impinging on the calorimeter, on the geometry and on the strength of the magnetic field. For this effect, the specific orientation of the magnetic field relative to the absorber plates is thus important and is different for HB and HE. At the LHC, the directions of particles emitted from the IP are essentially normal to the scintillator plane, the magnetic field direction is parallel to the scintillator plane in $\mathrm{HB}$ and perpendicular in HE. Previous measurements [15] and this study showed that the light yield increase due to the magnetic field is the same for muons and for particles from radioactive sources, while another complementary study [15] showed that the effect is different for pions and electrons, as electrons produce more knock-on electrons than pions. There is a third effect due to the HPD geometry. When a muon passes through a single tower, all the photons impinge on one single HPD pixel. However, the electrons from the photocathode spread out and up to $2 \%$ of the signal is measured in neighboring pixels, a phenomenon called cross talk. The magnetic field helps in reducing this cross talk, but any misalignment of the HPD axis with respect to the magnetic field axis increases the cross talk.
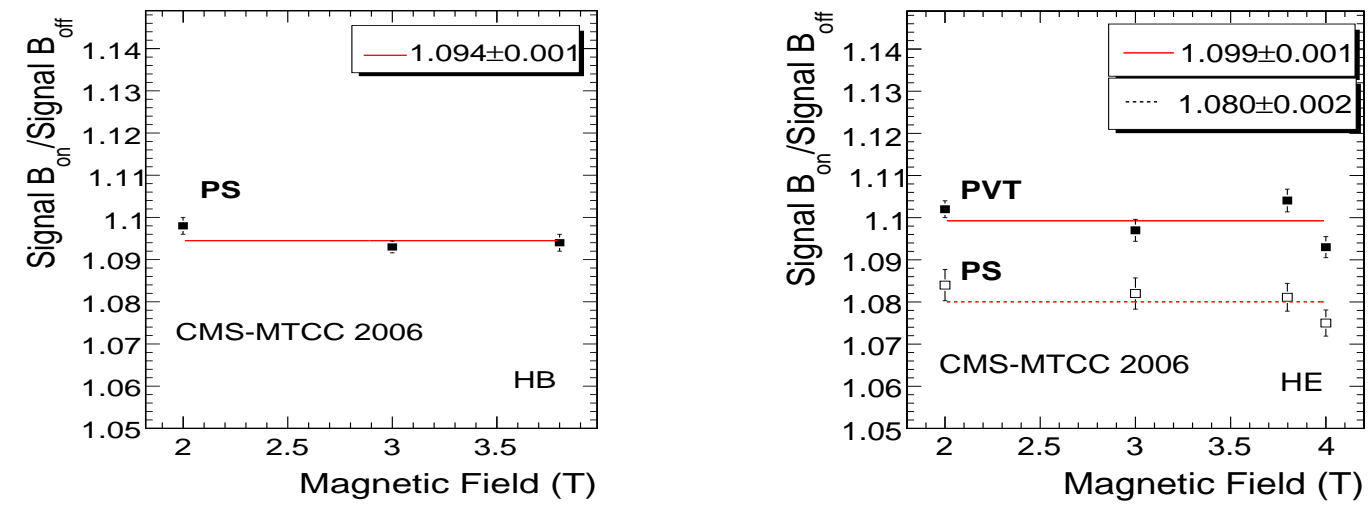

Figure 3: Ratio of the scintillator signals in a single layer with magnetic field on and off, measured using a radioactive source during MTCC. The left figure is for polystyrene (PS) in HB and the right figure is PVT (filled squares) and PS (open squares) in HE. The horizontal lines represent the averages of each data set. The difference for PS between HB and HE is due to the curling of the knock-on electrons and the magnetic field direction.

The magnetic field effect on the HCAL was observed by comparing the measurements performed with a moving radioactive wire source, with field on and with field off during the MTCC. The inners layer (Layer 0) of HB and HE and all layers of $\mathrm{HO}$ are made of $1 \mathrm{~cm}$ thick polymer polyvinyl toluene (PVT); all other layers of $\mathrm{HB}$ and $\mathrm{HE}$ are made of $0.4 \mathrm{~cm}$ thick polymer polystyrene(PS). Figure 3 shows the measurements performed at several magnetic field values for a typical polystyrene layer of $\mathrm{HB}$ (left) and for two layers of $\mathrm{HE}$ (right), one PVT and one PS. The PVT layer of HB was not installed during MTCC. The magnetic field effect on PS of $9.4 \%$ measured in HB during MTCC agrees well with the expected value. The $2 \%$ difference in 
brightening between PVT and PS, shown in Fig. 3(right), was also observed in an independent study [15]. For HE, a signal enhancement of about $6 \%$ is due to the increased brighteness of the polymer polystyrene, and the rest is due to the path length of the knock-on electrons. The comparison of CRAFT and CRUZET data reveals a net signal enhancement at 3.8 T of $1.090 \pm 0.005$ for HB and $1.084 \pm 0.013$ for HE, consistent with the MTCC measurements of $1.094 \pm 0.001$ for $\mathrm{HB}$ and $1.080 \pm 0.002$ for HE.

\subsection{The Outer Hadron Calorimeter}

All scintillator layers in $\mathrm{HO}$ are made of $1 \mathrm{~cm}$ thick PVT. Ring 0 has two scintillator layers, while Rings 1 and 2 have a single scintillator. Figure 4 (left) shows the field strength at the position where the scintillators are located. The first scintillator layer of Ring 0 , at a radius of $3.82 \mathrm{~m}$, is located in a region with a very small magnetic field. The signals in the two layers in Ring 0 are added optically and the brightening effect is the average of the effects in the two scintillator layers. The other scintillator layers are at a radial distance of $4.07 \mathrm{~m}$ from the beam line, where the magnetic field is more important. The scintillator brightening for Ring 0 is about $1 \%$, and for Rings 1 and 2 it varies between $2 \%$ and $4 \%$. This is to be compared to the $8-10 \%$ effect observed in $\mathrm{HB}$ and HE above $2 \mathrm{~T}$.
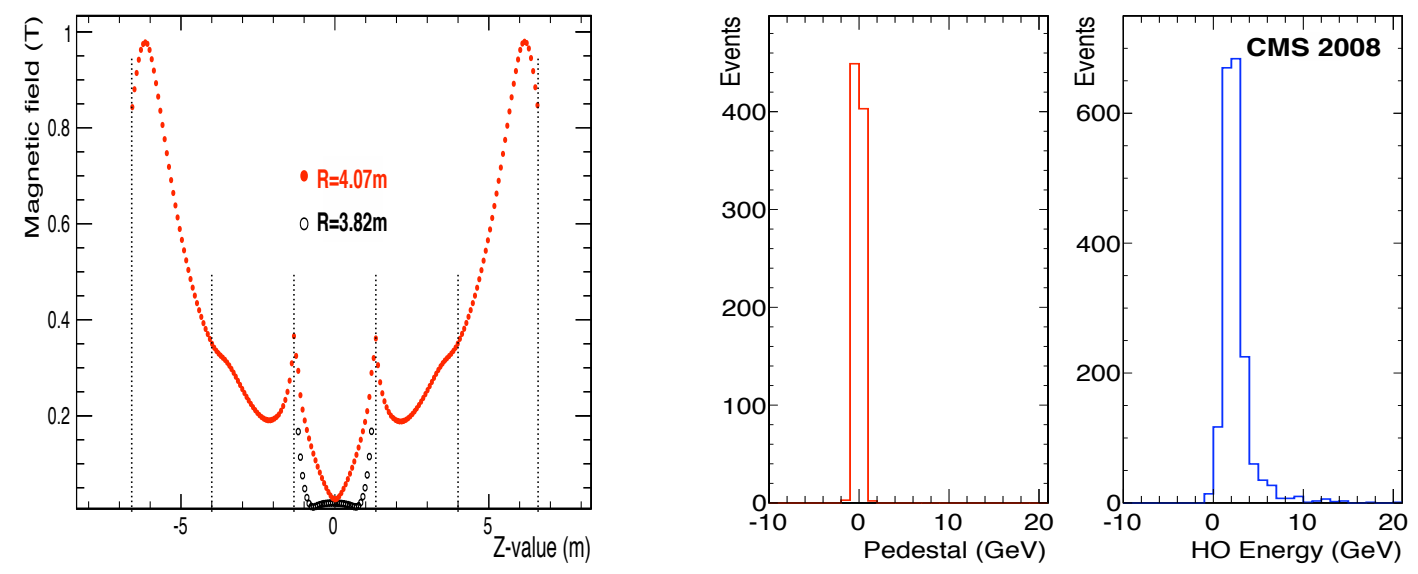

Figure 4: Left: Magnetic field strength at the location of the HO scintillators, as calculated with the TOSCA program [16]. The vertical lines indicate the ring borders. Ring 0 has two scintillator layers, at radii $3.82 \mathrm{~m}$ and $4.07 \mathrm{~m}$, respectively. Right: Pedestal and cosmic ray muon signal in Ring 0 of $\mathrm{HO}$.

Cosmic ray muon signals are observed in all rings of HO. In Ring 0 , with two scintillator layers, the signals are twice as large as in Rings 1 and 2. Figure 4 (right) shows the distributions of the pedestal and the pedestal subtracted muon signal in Ring 0 .

\subsection{The Forward Hadron Calorimeter}

The magnetic field strength in the HF region is significantly reduced compared to that in the $\mathrm{HB}$ and HE regions, allowing the use of conventional PMTs.

During CRAFT, the magnetic field at the PMT locations was measured to be less than $0.03 \mathrm{~T}$, and proper PMT operation was verified at full magnet current. Each PMT is shielded with individual tubes made of a nickel-iron alloy and of soft iron. HF was fully operational and stable during CRAFT and the LHC startup. 
In the CMS cavern, the number of cosmic ray muons decreases significantly with increasing incidence angle relative to the vertical direction. Therefore, it was not possible to validate the calibration of HF channels with the CRAFT data, due to the lack of muons in the horizontal direction.

The LHC startup data from September 2008, however, proved useful in validating the calibration constants, and will be discussed in Section 5 . Occasional abnormally large signals were found in individual HF channels during the LHC startup. The signals were found to be due to muons striking directly the window faces of individual PMTs. Algorithms for rejecting such abnormal signals, by comparing the energies reconstructed from long and short fibers with the same $(\eta, \phi)$ values, were subsequently developed based on these data [17]. The LHC beam halo data show that the current algorithms can reduce the rate of such abnormal signals by a factor of one thousand.

\section{Optimization of the HCAL Operation using CRAFT Data}

This section describes the information obtained from CRAFT about noise levels, detector stability and optimization of hardware settings, like high voltage and zero-suppression parameters, which could influence the HCAL energy resolution.

The HCAL has a total of 2592 channels in HB, 2592 in HE, 1728 in HF and 2160 in HO. At the beginning of CRAFT, $99.3 \%$ of $\mathrm{HB}, \mathrm{HE}$ and $\mathrm{HF}$, and $95.5 \%$ of $\mathrm{HO}$ channels were operational. About $75 \%$ of the non-operating channels had photodetector (HPD or PMT) problems and the rest had electronics failures of various types.

Several HPDs, about $10 \%$ of the total, functioned poorly when the magnetic field reached full strength. The high voltage for those channels was either reduced by $1.5 \mathrm{kV}$, leading to a $~ 30 \%$ reduction in gain, or was completely turned off. After this action was taken, $87 \%$ of the HCAL was operating at the nominal high voltage of $7.5 \mathrm{kV}$ both for the barrel and the endcaps. No evidence of further degradation of HPD functioning was observed during CRAFT. In HF, which employs PMTs, the high voltage was set at $1.25 \mathrm{kV}$ as originally planned.

The problematic HPDs were replaced after CRAFT. As of September 2009, all the channels in the barrel, endcap and forward regions are operational at full magnetic field, while the number of non-operational channels in $\mathrm{HO}$ is at the level of $2 \%$. As a precaution, the nominal high voltage during LHC collisions will be set $0.5 \mathrm{kV}$ lower than in CRAFT for the barrel and the endcaps, at a cost of a $12 \%$ reduction in gain. The impact on jet energy resolution is small, of the order of $5 \%$.

\subsection{Performance of Photodetectors and Impact on the Trigger}

During CRAFT, the rate of HPD noise replicating an energy deposit of $20 \mathrm{GeV}$ or more in a single channel was below $0.2 \mathrm{~Hz}$ [17], after the malfunctioning HPDs were excluded. Figure 5 shows the measured total noise rate for all trigger towers in the barrel and the endcaps, as a function of the energy threshold. The signals of the longitudinal segments of a tower are added to form a trigger tower in HB, in HB-HE interface region and in HE (Fig. 1). Three sources contribute to this noise rate [17]: (a) the ion feedback inside the HPD (two to three neighboring HPD pixels with energy greater than $1 \mathrm{GeV}$ ), which affects mainly single channels, (b) the noise from the whole HPD, which affects 18 channels simultaneously (all HPD pixels with energy greater than $1 \mathrm{GeV}$ ), and (c) coherent noise in a readout box (RBX), which affects a full set of 72 channels (all channels with energy greater than $1 \mathrm{GeV}$ ); one readout box includes the signals from four HPDs. The ion feedback is predominantly caused by a thermally emitted 
electron that ionizes the residual gas inside the HPD acceleration gap. The ion is accelerated back to the cathode and liberates further electrons, causing a signal equivalent to many photoelectrons. Misalignment of the HPD axis with respect to the axis of the solenoidal field can reduce the voltage of the HPD above which electrical discharges can occur. This can lead to an avalanche of secondary electrons, producing significant energy deposition in a large number of channels within an HPD. This is the main noise contribution above $100 \mathrm{GeV}$ and is referred to as "HPD discharge". The cause of the readout box coherent noise is not yet understood, but measurements show that it does not have an impact on the trigger and can be easily identified during the event reconstruction.

The HPD noise, with or without magnetic field, has a small impact on the total trigger rate. For example, without magnetic field and with a threshold of $10 \mathrm{GeV}$, the HCAL self-trigger rate is $130 \mathrm{~Hz}$; at full field the rate increases by $40 \mathrm{~Hz}$. This is to be compared with the $100 \mathrm{kHz}$ trigger rate expected during collisions. Furthermore, the rate decreases rapidly as a function of the applied threshold, as seen in Fig. 5 .

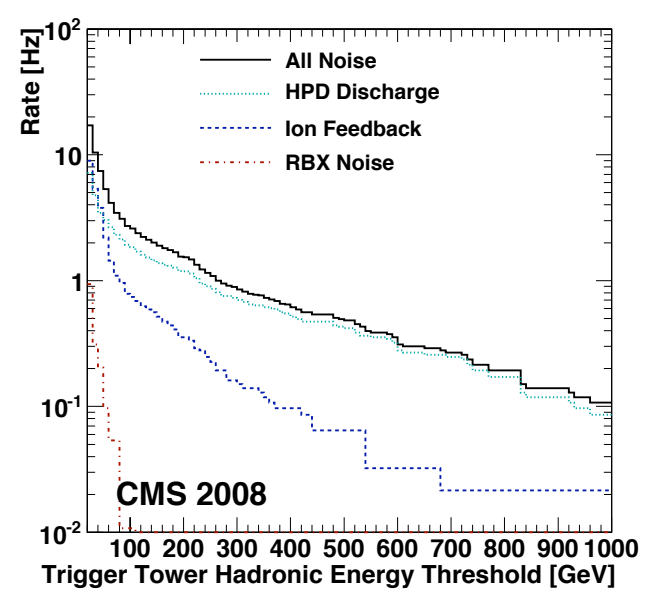

Figure 5: Total noise rate for around 4000 trigger towers as a function of the energy threshold, during CRAFT with the magnet at full field. A minimum energy of $20 \mathrm{GeV}$ was required in at least one HB or HE readout box (RBX). About $280 \mathrm{HPDs}$, corresponding to 5040 channels of $\mathrm{HB}$ and HE, are included in the analysis; HPDs that were replaced in the beginning of 2009 are excluded.

Special triggers to monitor the HPD noise rate, offline software to identify the corresponding atypical signals at the event reconstruction level [17], and the corresponding simulation tools have been developed and are available. Initial tests on data show that the algorithms for identifying noise and problematic channels correctly mark both persistent and sporadic known problems with high efficiency.

\subsection{Performance of Readout Electronics and Impact on Energy Thresholds}

The electronic noise and the pedestals in the QIE chips were found to be stable during CRAFT. The QIE chips read the signal over four time intervals of $25 \mathrm{~ns}$ each, using four pipelinechannels (numbered 0 to 3), and output four ADC values; the cycle repeats every $100 \mathrm{~ns}$. For each QIE one measures four pedestal values of a few ADC counts each (one for each $25 \mathrm{~ns}$ time interval), which are subtracted from the corresponding signal. The left plot in Fig. 6 shows the values of the four pedestals and their averages for a particular HB tower, over a period of ten 
days. There is a small variation of the individual pipeline-channel pedestals over time, with a strong anti-correlation among them. The average of the four pedestals does not change over this time period.
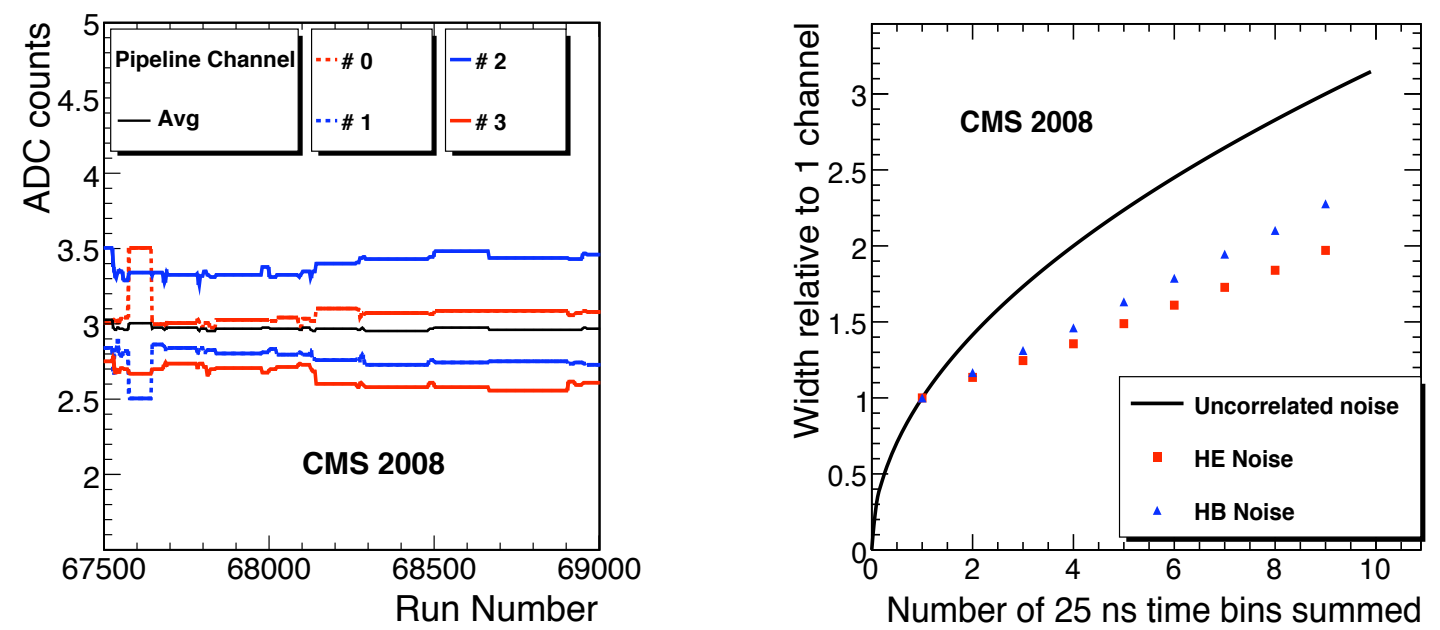

Figure 6: Left: The four pedestal values corresponding to the four $25 \mathrm{~ns}$ channels and their average, for one $\mathrm{HB}$ calorimeter tower over a period of 10 days. Right: The measured pedestal width in HB and HE when averaging over several (1 to 9) time slices (TS). The solid line represents the expected noise $(\propto \sqrt{n})$ in the absence of correlations. The signal reconstruction will sum four TS at low luminosity and two TS at high luminosity. The single channel noise at $7.5 \mathrm{kV}$ for $1 \mathrm{TS}$ is 140 and $200 \mathrm{MeV}$ for $\mathrm{HB}$ and $\mathrm{HE}$, respectively.

The four pedestal values are correlated due to the integration of the pulse shaper, as demonstrated in the right plot of Fig. 6. This figure shows the observed noise averaged over a variable number $n$ (from 1 to 9 ) of $25 \mathrm{~ns}$ time periods, compared to the increase as $\sqrt{n}$ expected in the absence of correlations. These noise correlations have been included in the HCAL simulation.

The width of the pedestal distribution allows one to estimate the QIE electronic noise. For example, the measured electronic noise during CRAFT in a single time slice corresponds to an equivalent energy in a tower of $140 \mathrm{MeV}$ for $\mathrm{HB}, 200 \mathrm{MeV}$ for $\mathrm{HE}, 280 \mathrm{MeV}$ for $\mathrm{HO}$ and $370 \mathrm{MeV}$ for $\mathrm{HF}$ (the larger HF noise remains small compared to the energy corresponding to a single photoelectron, $4 \mathrm{GeV}$, and to the typical energies of particles impinging $\mathrm{HF}$ ). The increase when integrating over more than one time slice is modest, as can be inferred from Fig. 66(right).

The HCAL information obtained from a tower is not recorded if the signal is below a threshold, that is, the HCAL data are "zero-suppressed". The information of a tower is kept only if the sum of ADC counts in two adjacent time slices is larger than 2 counts above pedestal for $\mathrm{HB}, 3$ counts for $\mathrm{HE} / \mathrm{HO}$, and 4 counts for HF. After taking into account the final high voltage settings planned for collision data, these thresholds correspond to $\sim 400 \mathrm{MeV}$ for $\mathrm{HB}$, $\sim 700 \mathrm{MeV}$ for $\mathrm{HE}, \sim 1 \mathrm{GeV}$ for Ring 0 of $\mathrm{HO}$, and $\sim 1 \mathrm{GeV}$ for HF. These threshold values were selected such that the HCAL occupancy is reduced to about $20 \%$ at the nominal LHC luminosity. Since some towers, which contain little energy, are eliminated by zero-suppression, this can reduce the measurement accuracy. However, since the energy thresholds for zero-suppression are well below the energy threshold applied in the jet reconstruction, and since a shower is distributed over many towers, zero-suppression will have little effect on jet and single high energy hadron energy measurements. It can, however, affect the HCAL energy measurement for low-momentum muons $\left(p_{\mu}<10 \mathrm{GeV} / c\right)$ traversing a single tower and all muons traversing 
multiple towers.

\section{Calibrations using Cosmic Ray Muons and LHC Beams}

The calibration of the HCAL has been performed using several techniques. First, test beam setups were used to determine, for parts of the HCAL subsystems, the detector response to radioactive sources and to electrons, muons and hadrons of known momenta. Then, the intercalibrations of all channels in $\mathrm{HB}, \mathrm{HE}$, and HF were performed with radioactive sources mounted on moving wires, exactly as was done in the test beam setups. These source measurements also propagate the absolute energy scale for each subsystem. The energy scale for the reference $\mathrm{Co}^{60}$ source was made with respect to $50 \mathrm{GeV} / c$ pions in the case of $\mathrm{HB}$ and $\mathrm{HE}$, while for $\mathrm{HF} 100 \mathrm{GeV} / \mathrm{c}$ electrons were used.

A total of 31 beam splash events from the beam arriving from the $+z$ side and 35 events from the $-z$ side were recorded during the 2008 LHC commissioning exercise, with the CMS magnetic field off. In these events, muons from secondary decays penetrated the detector. Since the scintillator planes of $\mathrm{HB}$ and $\mathrm{HO}$ are parallel to the beam line, the muons from splash events traverse the full length of the scintillators giving a very large signal. The splash events could not be used to study the absolute energy scale calibration because their energy and muon flux is not accurately known. However, these events were helpful for measuring the relative scale between $\phi$ sectors and between the $\pm z$ halves of HB and the two HE endcaps.

The initial calibration obtained from measurements with radioactive sources was tested, and in some cases improved, using cosmic ray muons and the beam splash events for a significant fraction of HB channels. However, since cosmic ray muons in the CMS cavern are essentially vertical, $\mathrm{HB} \phi$ sectors in the horizontal plane near $\phi=0^{\circ}$ (sectors $71,72,1$ to 4 ) and near $\phi=180^{\circ}$ (sectors 33 to 38) were not included in this study, while in $\mathrm{HO}$ all channels were calibrated using cosmic ray muons. All channels in HE and HF were mainly tested with beam splash events.

To ensure good energy measurement, only events collected without zero-suppression are used in the calibration studies.

\subsection{The Barrel and Endcap Hadron Calorimeters}

All HB and HE scintillator tiles had their response measured in-situ using either 1.5 or $5 \mathrm{mCi}$ $\mathrm{Co}^{60}$ sources. The averages of the signals in the 17 layers of $\mathrm{HB}$ and HE were used to equalize the response of all HB and HE towers. The absolute energy scale was set using the response to $50 \mathrm{GeV} / c$ charged pions of the parts of the detector exposed to the test beam studies.

Table 1: Mean and RMS of the muon energy distributions measured in four regions of $\mathrm{HB}$, before and after correcting the calibration constants using cosmic ray muon data information.

\begin{tabular}{|l|c|c|c|c|}
\hline $\begin{array}{l}\text { Detector region } \\
\text { region }\end{array}$ & $\begin{array}{c}\text { Mean before } \\
\text { correction }(\mathrm{GeV})\end{array}$ & $\begin{array}{c}\text { RMS before } \\
\text { correction }(\mathrm{GeV})\end{array}$ & $\begin{array}{c}\text { Mean after } \\
\text { correction }(\mathrm{GeV})\end{array}$ & $\begin{array}{c}\text { RMS after } \\
\text { correction }(\mathrm{GeV})\end{array}$ \\
\hline HB-: $i \phi 5-32$ & $1.87 \pm 0.03$ & 0.12 & $1.78 \pm 0.01$ & 0.05 \\
HB-: $i \phi 39-70$ & $1.83 \pm 0.02$ & 0.08 & $1.75 \pm 0.02$ & 0.07 \\
HB+: $i \phi 5-32$ & $1.81 \pm 0.02$ & 0.08 & $1.78 \pm 0.01$ & 0.05 \\
HB+: $i \phi 39-70$ & $1.70 \pm 0.02$ & 0.09 & $1.74 \pm 0.01$ & 0.04 \\
\hline
\end{tabular}

The cosmic ray muons and splash events were used to check the initial calibration conditions. The results from both methods point to non-uniformities in $\mathrm{HB}$ calorimeter response in differ- 

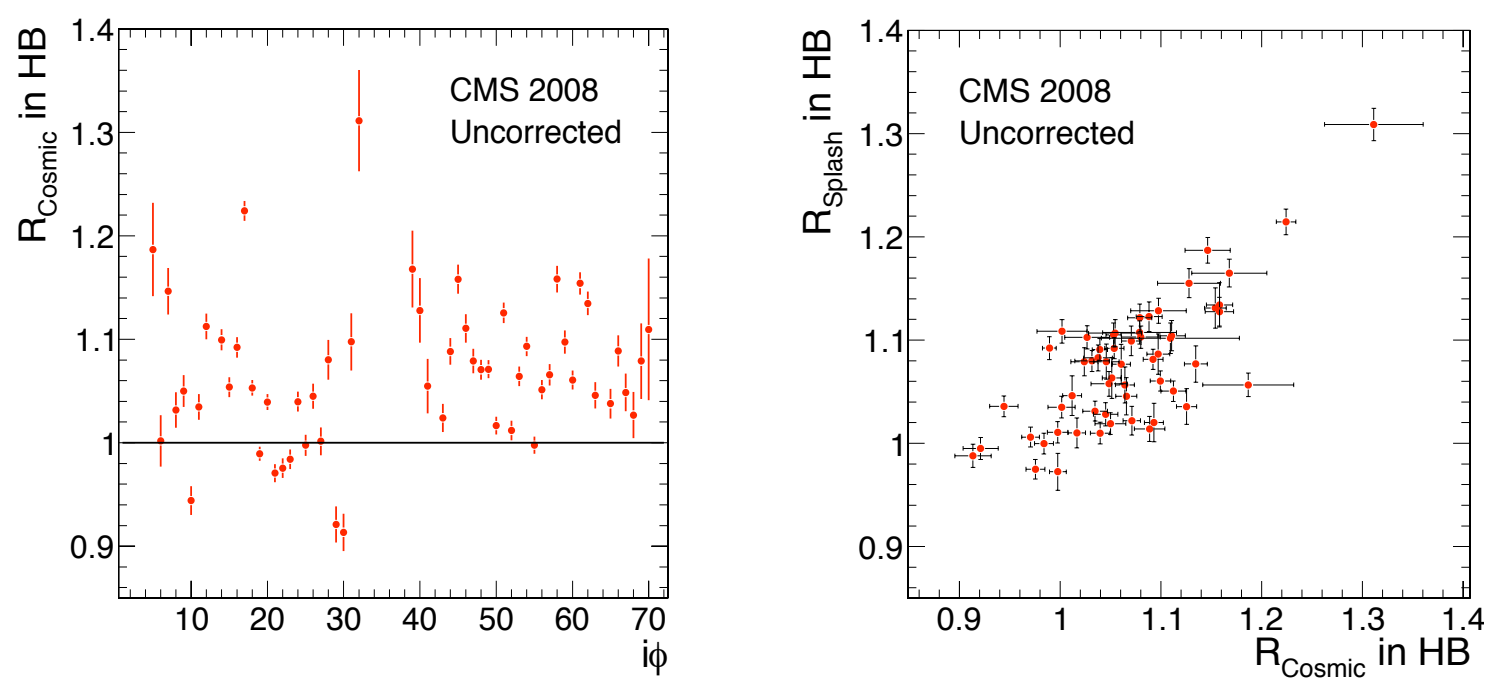

Figure 7: Left: Ratio, for cosmic ray muons, of the average signal amplitudes in the two halves $(+z$ and $-z)$ of $\mathrm{HB}$, as a function of the $\phi$ sector index, $i \phi$, after correcting the average signal for the muon path length and normalizing to the thickness of $\mathrm{HB}$ at $\eta=0$. Right: Correlation between cosmic ray muon and splash muon signals, for the ratio of the average signal in the two halves of HB. The splash muon signals were corrected for the asymmetry in the muon flux by comparing the events from the beam arriving from the $+z$ side and the $-z$ side.

ent $\phi$ sectors, and to a difference in the energy scale between the two halves of HB. Figure 7 (left) shows the ratio of the muon signal amplitudes in HB for $z<0$ and $z>0$ for cosmic ray muon events, $R_{\text {Cosmic }}$ as a function of the $\phi$ sector index, $i \phi$. While Fig. 7 (right) shows that measurements for this ratio based on cosmic ray muons and beam splash events, $R_{\text {Splash }}$, have a $75 \%$ correlation. The average ratio deviates from unity by $\sim 7 \%$ with a sector-by-sector spread of about $10 \%$ (see Table 1). The source of these differences is not understood at this time.

The results from the cosmic ray muons were used to correct the calibration of the various HPDs, with one single parameter applied to all channels contained in a given $\phi$ sector of one of the two $\pm z$ halves of HB. Only muons traversing a single $\phi$ sector and less than five $\eta$ channels were used in the analysis. About half a million CRAFT muons with momentum below $100 \mathrm{GeV} / \mathrm{c}$ remain after the full event selection. Sector 13 of HB, where an HPD was replaced before CRAFT, was excluded from these studies. Figure 8 shows the peak value of the distribution of the average energy deposited in HB by cosmic ray muons, averaged over all $\eta$ values of a given $\phi$ sector for two independent data samples. The plot on the left shows the results for the data used to determine the corrections, while the plot on the right shows the results after the corrections are applied to a smaller but independent data sample. Table 1 presents the corresponding results for four HB regions. After corrections, the signal spread is smaller and the differences between the mean values for the four regions of $\mathrm{HB}$ is significantly reduced, reflecting the improvement of the HPD intercalibration. The mean muon energy measured in this analysis is smaller than the one shown in Fig. 2 because only tracks with a momentum below $100 \mathrm{GeV} / c$ were used.

Similar studies were performed for HE with a limited number of cosmic ray muon events and all splash events. The ratio of the muon signals for the two endcaps as a function of the $\phi$ sector 

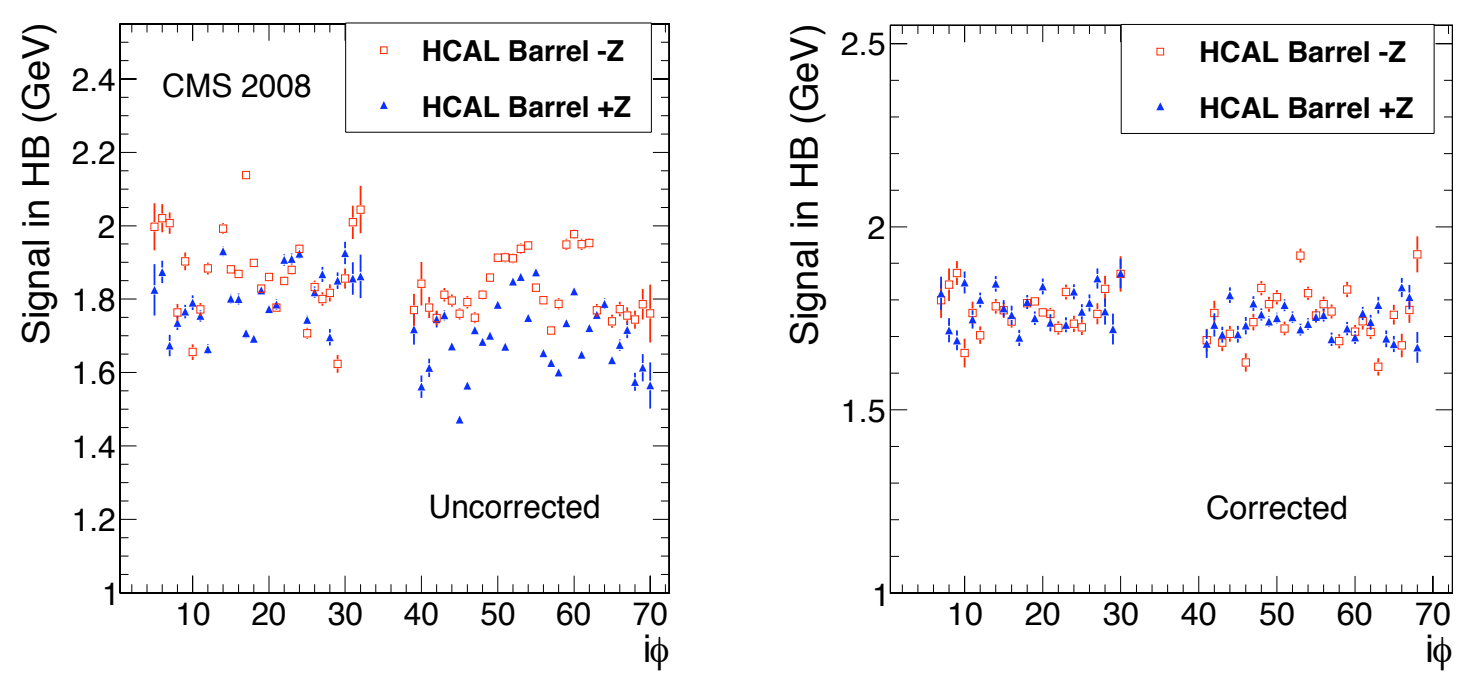

Figure 8: Cosmic ray muon signal in HB averaged over all $\eta$ values for each $\phi$ sector, $i \phi$, before (left) corrections for a sample of $\sim 400$ thousand events and after (right) corrections for sample of $\sim 80$ thousand events. The triangles (open squares) represent the results for the $+z(-z)$ side of the detector. The mean and RMS values for the muon signals for four HB regions are presented in Table1.
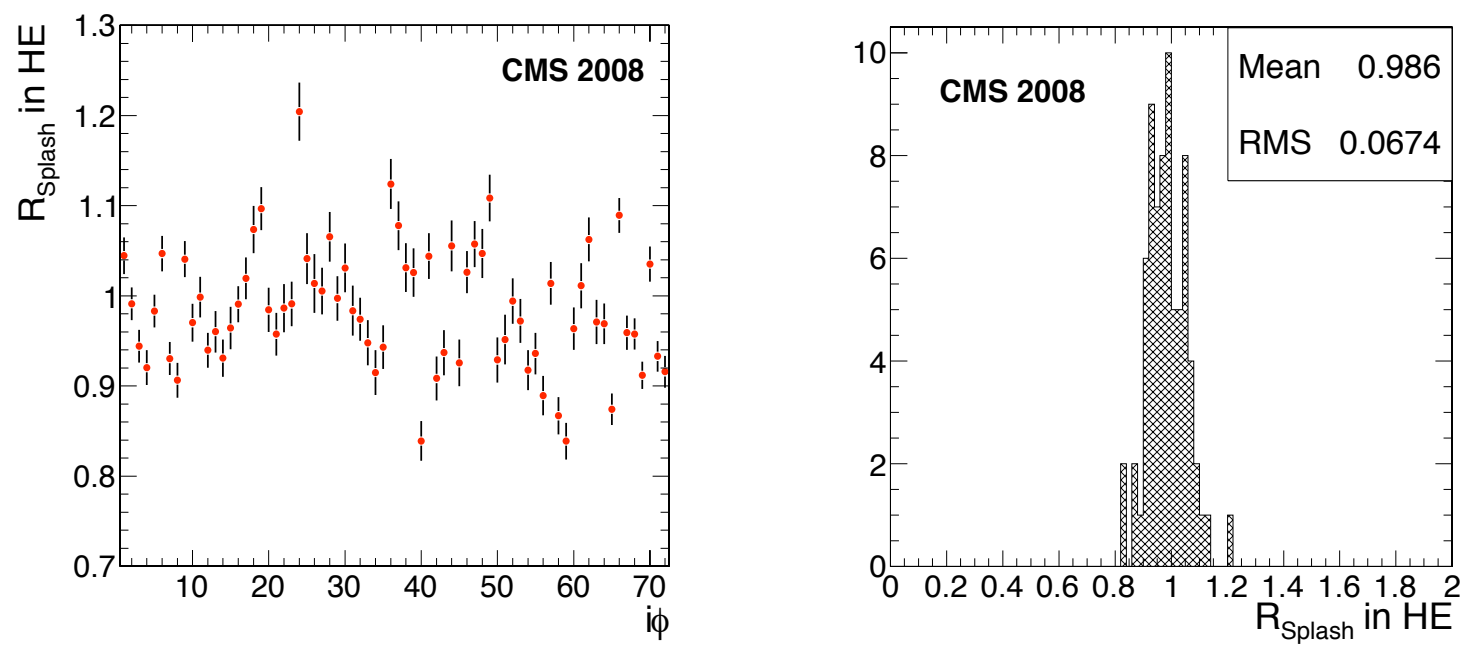

Figure 9: Left: Ratio, of the splash muons, of the average signal amplitudes in the two HCAL endcaps, $-z$ and $+z$, as a function of the $\phi$ sector index, $i \phi$. Right: Corresponding distribution of the signal ratios. 
index is shown in Fig. 9 (right) for splash events. The $R_{\text {Splash }}$ mean and the spread of this ratio are shown in Fig. 9(left). The average is consistent with unity and the spread is smaller than $7 \%$. Therefore, no correction to the HE calibration was made.
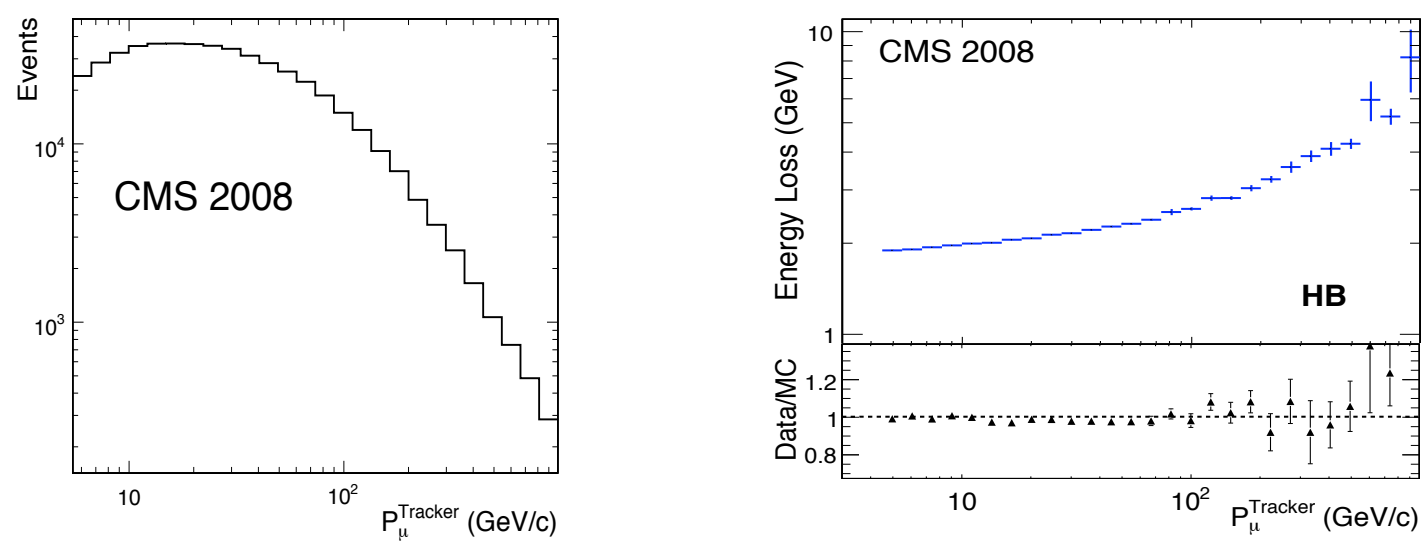

Figure 10: Left: Momentum spectrum of the muons selected by this analysis. Right: (top) Energy loss of cosmic ray muons in HB after correcting the signal mean value for the muon path length and normalizing to the thickness of $\mathrm{HB}$ at $\eta=0$; (bottom) ratio of data and Monte Carlo simulation predictions (arbitrary scale) for the bottom half of the calorimeter.

Cosmic ray muons were also used to validate the absolute energy scale and the material description in HB and HE simulations [18]. The absolute energy scale was tested by comparing the energy loss of muons measured at a test beam and in CRAFT, at a fixed muon momentum. From the test beam data it was derived that $150 \mathrm{GeV} / \mathrm{c}$ muons deposited $2.80 \pm 0.03 \mathrm{GeV}$ in HB [6]. The energy loss measured from CRAFT data, after taking into account the magnetic field effects and the muon path length, is $2.85 \mathrm{GeV}$ with a statistical error of $0.02 \mathrm{GeV}$ for the momentum range between 135 and $170 \mathrm{GeV} / c$. This measurement is in good agreement with the test beam result. A wide muon momentum range was used to test the simulation. Figure 10(left) shows the cosmic ray muon energy spectrum measured with the tracker. Figure 10 (right top) shows the muon energy deposited in a calorimeter tower, corrected for the path length and normalized to the thickness of $\mathrm{HB}$ at $\eta=0$, that is, $110 \mathrm{~cm}$. The relativistic rise of the energy loss for muons between 6 and $1000 \mathrm{GeV} / c$ is clearly observed and is consistent with expectations [19]. Figure 10 (right bottom) illustrates the consistency of the data and simulation [18] as a function of the track momentum; the data to simulation ratio shown at an arbitrary scale.

\subsection{The Outer Hadron Calorimeter}

Since the HO scintillators were not precalibrated with the radioactive source, the calibration of each tower was performed using cosmic ray muons from CRUZET, at a uniform high voltage value of $8 \mathrm{kV}$. This calibration agrees well with the measurements performed at a test beam [6].

Figure 11 shows the mean values of the muon signals in $\mathrm{HO}$, given in ADC counts for CRUZET data. The structure within each ring reflects the differences in signal attenuation caused by the varying length of the fibers that carry the signals to the photodetectors. 


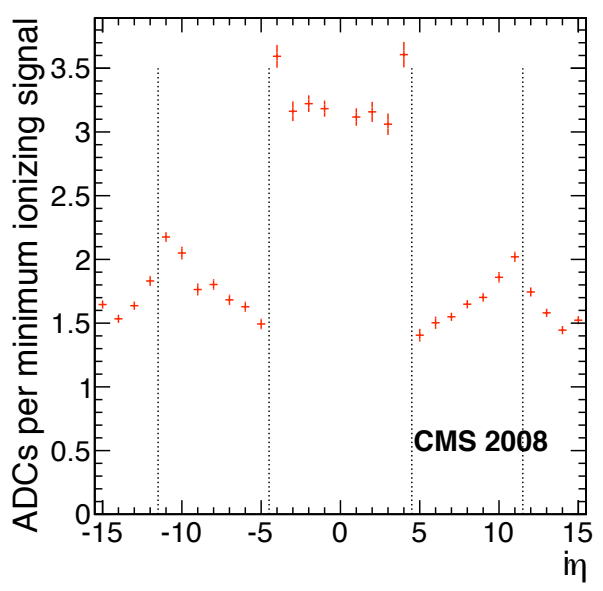

Figure 11: Mean values of the signals measured for a minimum ionizing muon in $\mathrm{HO}$ as a function of the $\eta$ index, $i \eta$. The vertical lines indicate the borders of the rings. The differences in signal size between Ring 0 and Rings 1 or 2 are due to the fact that Ring 0 contains two layers of scintillators, for which the signals are added optically. The structure reflects the differences in the fiber lengths.

\subsection{The Forward Hadron Calorimeter}

The calibration for HF was obtained from measurements with radioactive sources and from test beam data. Five HF wedges were precalibrated at the test beam with $100 \mathrm{GeV} / c$ electrons [3] and with a $5 \mathrm{mCi} \mathrm{Co}^{60}$ source. Figure 12 displays the good correlation between the signals of the electrons and the source measurement in the same $\eta, \phi$ sector, separately for short (crosses) and long (circles) fibers. The average value for the calibration coefficients of the short (long) fibers is $0.34(0.21) \mathrm{GeV} / \mathrm{fC}$. This information was used to set the energy scale for all the channels which were not exposed to the beam, using the results of their measurement with the radioactive source.

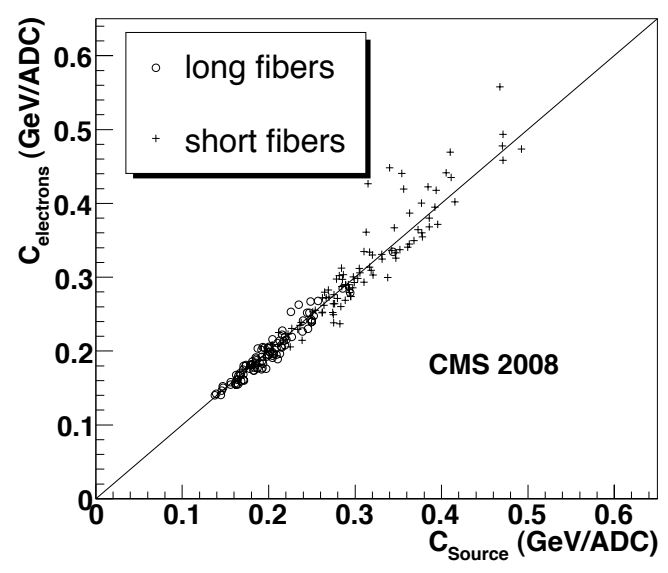

Figure 12: The signal in $\mathrm{GeV} / \mathrm{ADC}$ for $100 \mathrm{GeV} / c$ electrons from the test beam (vertical axis) versus the radioactive source in $\mathrm{GeV} / \mathrm{ADC}$ for the same $\eta, \phi$ space, for short (crosses) and long (circles) fibers for HF+ and HF-. This information was used to set the energy scale of the calibration obtained with the radioactive source method.

The monitoring of the HF calibration relies on single photoelectron distributions (SPE) mea- 
suments performed with the LED system. Earlier measurements performed at a test beam showed that 25 SPEs are equivalent to $100 \mathrm{GeV}$ [3]. The major difference between using the SPE measurements and using a radioactive source is that the SPE characterizes the PMT performance alone, while the radioactive source creates Cherenkov light inside the detector which in turn detected by the PMTs. Therefore, the light collection efficiency and the PMT photocathode sensitivity are taken into account in the source calibration, but not in the SPE technique. However, if the attenuation of Cherenkov light as it propagates from the fiber to the PMT is only affected by small variations from channel to channel, or if the attenuation is negligible, the two calibration techniques should be equivalent.
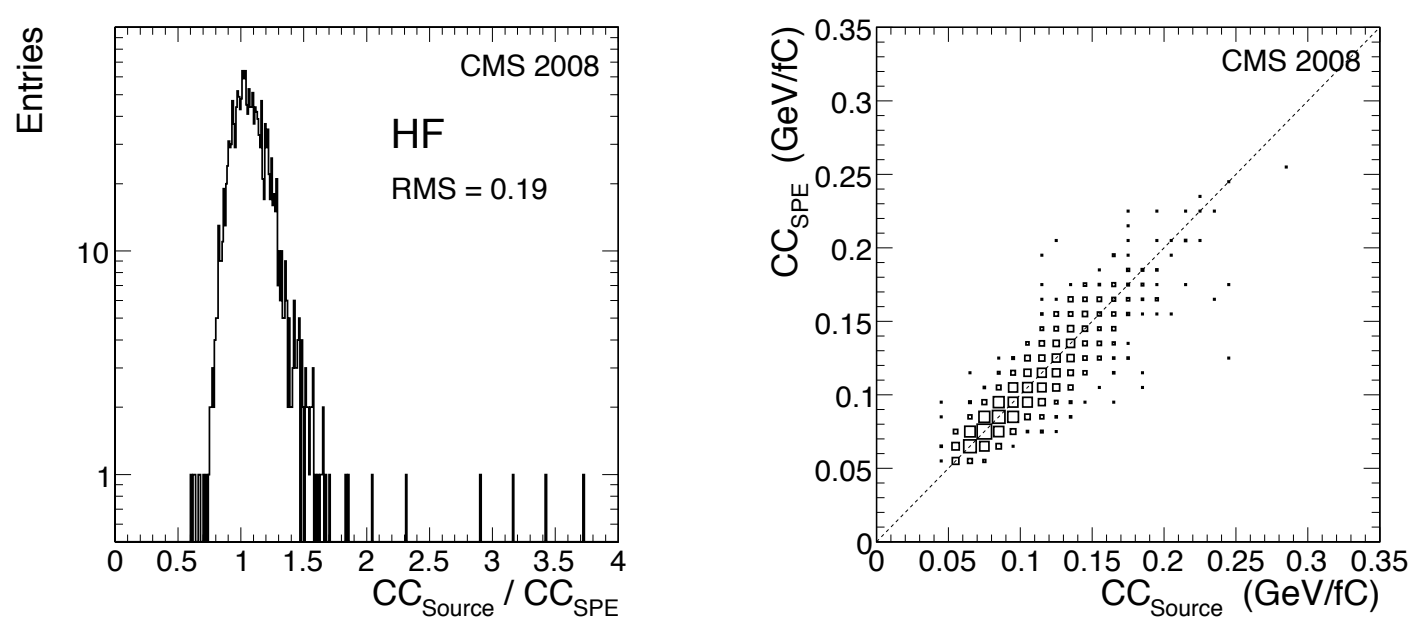

Figure 13: The calibration coefficients (CC) for HF+ and HF- obtained with the SPE (single photoelectron) method are compared to the standard values obtained with the radioactive source method before (left plot shows ratio) and after (right plot shows correlation) correcting the calibration, see text. The ratio of the two sets of constants is around 1.1 because the measurements were performed with a different HV setup.

A comparison between the results obtained with the radioactive source and new SPE measurements, performed after the final HF installation, revealed that 20 of the 1728 channels had inconsistent calibration, see Fig. 13 (left). The beam splash events confirmed this calibration problem since the same channels were found to show an energy response inconsistent with their neighbors. Therefore, for those channels the source calibration constants were replaced by the SPE constants.

Figure 13 (right) shows the correlation between the calibration coefficients obtained from single photoelectron distributions and the coefficients obtained from the radioactive source calibration, after correcting the few outliers mentioned above.

\section{Summary}

The performance of the CMS hadron calorimeter has been studied using cosmic ray and beam splash events, providing improved calibrations with respect to those obtained in test beam data and with radioactive sources.

As a result of this calibration effort, the barrel portion is intercalibrated to the level of $5 \%$, for $85 \%$ of the channels. The other HCAL portions have all their channels intercalibrated to better than $10 \%(\mathrm{HE}), 12 \%(\mathrm{HF})$ and $5 \%(\mathrm{HO})$. Furthermore, the presence of the magnetic field results 
in a measured increase of the signal, by $9.4 \%$ in $\mathrm{HB}$ and $8 \%$ in $\mathrm{HE}$, as expected.

Noise studies have been performed, showing that noise will contribute a trigger rate of around $100 \mathrm{~Hz}$. This rate is stable.

In conclusion, the CRAFT data and the beam splash events from 2008 provided an improved calibration of the HCAL. This subdetector is now ready for LHC collisions.

\section{Acknowledgments}

We thank the technical and administrative staff at CERN and other CMS Institutes, and acknowledge support from: FMSR (Austria); FNRS and FWO (Belgium); CNPq, CAPES, FAPERJ, and FAPESP (Brazil); MES (Bulgaria); CERN; CAS, MoST, and NSFC (China); COLCIENCIAS (Colombia); MSES (Croatia); RPF (Cyprus); Academy of Sciences and NICPB (Estonia); Academy of Finland, ME, and HIP (Finland); CEA and CNRS/IN2P3 (France); BMBF, DFG, and HGF (Germany); GSRT (Greece); OTKA and NKTH (Hungary); DAE and DST (India); IPM (Iran); SFI (Ireland); INFN (Italy); NRF (Korea); LAS (Lithuania); CINVESTAV, CONACYT, SEP, and UASLP-FAI (Mexico); PAEC (Pakistan); SCSR (Poland); FCT (Portugal); JINR (Armenia, Belarus, Georgia, Ukraine, Uzbekistan); MST and MAE (Russia); MSTDS (Serbia); MICINN and CPAN (Spain); Swiss Funding Agencies (Switzerland); NSC (Taipei); TUBITAK and TAEK (Turkey); STFC (United Kingdom); DOE and NSF (USA). Individuals have received support from the Marie-Curie IEF program (European Union); the Leventis Foundation; the A. P. Sloan Foundation; and the Alexander von Humboldt Foundation.

\section{References}

[1] CMS Collaboration, "The CMS Experiment at the CERN LHC", J. Inst. 3 S08004 (2008). doi:10.1088/1748-0221/3/08/s08004.

[2] L. Evans and P. Bryant (eds.), "LHC Machine”, J. Inst. 3 S08001 (2008). doi:10.1088/1748-0221/3/08/s08001.

[3] CMS-HCAL Collaboration, "Design, performance and calibration of the CMS forward calorimeter wedges", Eur. Phys. J. C53 (2008) 139.

doi:10.1140/epjc/s10052-007-0459-4.

[4] CMS-HCAL Collaboration, "Design, performance, and calibration of CMS hadron-barrel calorimeter wedges", Eur. Phys. J. C55 (2008) 159.

doi:10.1140/epjc/s10052-008-0573-y.

[5] CMS-HCAL Collaboration, "Design, performance, and calibration of the CMS hadron-outer calorimeter", Eur. Phys. J. C57 (2008) 653.

doi:10.1140/epjc/s10052-008-0756-6.

[6] CMS-HCAL Collaboration, "The CMS barrel calorimeter response to particle beam from 2 to $350 \mathrm{GeV} / \mathrm{c}^{\prime \prime}$, Eur. Phys. J. C60 (2009) 359.

doi:10.1140/epjc/s10052-009-0959-5.

[7] T. Christiansen, "The CMS magnet test and cosmic challenge", arXiv:0805.1882.

[8] CMS Collaboration, "Commissioning of the CMS Experiment and the Cosmic Run at Four Tesla", to be submitted to JINST (2009) arXiv:0911.4845. 
[9] CMS Collaboration, "Performance of CMS Hadron Calorimeter Timing and Synchronization using Cosmic Ray and LHC Beam Data", to be submitted to JINST (2009) arXiv:0911.4877.

[10] E. Hazen et al., "Radioactive source calibration technique for the CMS hadron calorimeter", Nucl. Instrum. Meth. A511 (2003) 311. doi:10.1016/S0168-9002(03)01971-5.

[11] S. Bertolucci et al., "Influence of magnetic fields on the response of acrylic scintillators", Nucl. Instr. Meth. A254 (1987) 561.doi:10.1016/0168-9002 (87) 90029-5.

[12] J. P. Cumulat, H. W. K. Cheung, J. Hassed et al., "Effects of magnetic fields on the light yield of scintillators", Nucl. Instrum. Meth. A293 (1990) 606. doi:10.1016/0168-9002(90)90331-Y.

[13] D. Blomker, U. Holm, R. Klanner et al., "Plastic scintillators in magnetic fields", Nucl. Instrum. Meth. A311 (1992) 505. doi:10.1016/0168-9002(92) 90648-N.

[14] J. Mainusch, F. Corriveau, R. Klanner et al., "Influence of magnetic fields on the response of a uranium scintillator sampling calorimeter", Nucl. Instrum. Meth. A312 (1992) 451. doi:10.1016/0168-9002(92)90194-9.

[15] M. Bertoldi et al., "Scintillators in magnetic fields up to 20-T", Nucl. Instrum. Meth. A386 (1997) 301.doi:10.1016/s0168-9002(96)01178-3.

[16] J. Simkin and C. W. Trowbridge, "Three-Dimensional Computer Program (TOSCA) for Nonlinear Electromagnetic Fields", technical report, CCLRC, 1979. RL-79-097.

[17] CMS Collaboration, "Identification and Filtering of Uncharacteristic Noise in the CMS Hadronic Calorimeter", submitted to JINST (2009) arXiv:0911.4881.

[18] S. Banerjee, "Readiness of CMS Simulation Towards LHC Startup", J. Phys. Conf. Ser. 119 (2008) 032006. doi:10.1088/1742-6596/119/3/032006.

[19] Particle Data Group, "Review of particle physics", Phys. Lett. B667 (2008) 1. doi:10.1016/j.physletb.2008.07.018, 


\section{A The CMS Collaboration}

Yerevan Physics Institute, Yerevan, Armenia

S. Chatrchyan, V. Khachatryan, A.M. Sirunyan

Institut für Hochenergiephysik der OeAW, Wien, Austria

W. Adam, B. Arnold, H. Bergauer, T. Bergauer, M. Dragicevic, M. Eichberger, J. Erö, M. Friedl, R. Frühwirth, V.M. Ghete, J. Hammer ${ }^{1}$, S. Hänsel, M. Hoch, N. Hörmann, J. Hrubec, M. Jeitler, G. Kasieczka, K. Kastner, M. Krammer, D. Liko, I. Magrans de Abril, I. Mikulec, F. Mittermayr, B. Neuherz, M. Oberegger, M. Padrta, M. Pernicka, H. Rohringer, S. Schmid, R. Schöfbeck, T. Schreiner, R. Stark, H. Steininger, J. Strauss, A. Taurok, F. Teischinger, T. Themel, D. Uhl, P. Wagner, W. Waltenberger, G. Walzel, E. Widl, C.-E. Wulz

National Centre for Particle and High Energy Physics, Minsk, Belarus

V. Chekhovsky, O. Dvornikov, I. Emeliantchik, A. Litomin, V. Makarenko, I. Marfin, V. Mossolov, N. Shumeiko, A. Solin, R. Stefanovitch, J. Suarez Gonzalez, A. Tikhonov

Research Institute for Nuclear Problems, Minsk, Belarus

A. Fedorov, A. Karneyeu, M. Korzhik, V. Panov, R. Zuyeuski

Research Institute of Applied Physical Problems, Minsk, Belarus

P. Kuchinsky

Universiteit Antwerpen, Antwerpen, Belgium

W. Beaumont, L. Benucci, M. Cardaci, E.A. De Wolf, E. Delmeire, D. Druzhkin, M. Hashemi, X. Janssen, T. Maes, L. Mucibello, S. Ochesanu, R. Rougny, M. Selvaggi, H. Van Haevermaet, P. Van Mechelen, N. Van Remortel

Vrije Universiteit Brussel, Brussel, Belgium

V. Adler, S. Beauceron, S. Blyweert, J. D'Hondt, S. De Weirdt, O. Devroede, J. Heyninck, A. Kalogeropoulos, J. Maes, M. Maes, M.U. Mozer, S. Tavernier, W. Van Doninck ${ }^{1}$, P. Van Mulders, I. Villella

Université Libre de Bruxelles, Bruxelles, Belgium

O. Bouhali, E.C. Chabert, O. Charaf, B. Clerbaux, G. De Lentdecker, V. Dero, S. Elgammal, A.P.R. Gay, G.H. Hammad, P.E. Marage, S. Rugovac, C. Vander Velde, P. Vanlaer, J. Wickens

Ghent University, Ghent, Belgium

M. Grunewald, B. Klein, A. Marinov, D. Ryckbosch, F. Thyssen, M. Tytgat, L. Vanelderen, P. Verwilligen

Université Catholique de Louvain, Louvain-la-Neuve, Belgium

S. Basegmez, G. Bruno, J. Caudron, C. Delaere, P. Demin, D. Favart, A. Giammanco, G. Grégoire, V. Lemaitre, O. Militaru, S. Ovyn, K. Piotrzkowski ${ }^{1}$, L. Quertenmont, N. Schul

Université de Mons, Mons, Belgium

N. Beliy, E. Daubie

Centro Brasileiro de Pesquisas Fisicas, Rio de Janeiro, Brazil

G.A. Alves, M.E. Pol, M.H.G. Souza

Universidade do Estado do Rio de Janeiro, Rio de Janeiro, Brazil

W. Carvalho, D. De Jesus Damiao, C. De Oliveira Martins, S. Fonseca De Souza, L. Mundim, V. Oguri, A. Santoro, S.M. Silva Do Amaral, A. Sznajder

Instituto de Fisica Teorica, Universidade Estadual Paulista, Sao Paulo, Brazil 
T.R. Fernandez Perez Tomei, M.A. Ferreira Dias, E. M. Gregores ${ }^{2}$, S.F. Novaes

Institute for Nuclear Research and Nuclear Energy, Sofia, Bulgaria

K. Abadjiev ${ }^{1}$, T. Anguelov, J. Damgov, N. Darmenov ${ }^{1}$, L. Dimitrov, V. Genchev ${ }^{1}$, P. Iaydjiev, S. Piperov, S. Stoykova, G. Sultanov, R. Trayanov, I. Vankov

University of Sofia, Sofia, Bulgaria

A. Dimitrov, M. Dyulendarova, V. Kozhuharov, L. Litov, E. Marinova, M. Mateev, B. Pavlov, P. Petkov, Z. Toteva ${ }^{1}$

Institute of High Energy Physics, Beijing, China

G.M. Chen, H.S. Chen, W. Guan, C.H. Jiang, D. Liang, B. Liu, X. Meng, J. Tao, J. Wang, Z. Wang, Z. Xue, Z. Zhang

State Key Lab. of Nucl. Phys. and Tech., Peking University, Beijing, China

Y. Ban, J. Cai, Y. Ge, S. Guo, Z. Hu, Y. Mao, S.J. Qian, H. Teng, B. Zhu

Universidad de Los Andes, Bogota, Colombia

C. Avila, M. Baquero Ruiz, C.A. Carrillo Montoya, A. Gomez, B. Gomez Moreno, A.A. Ocampo Rios, A.F. Osorio Oliveros, D. Reyes Romero, J.C. Sanabria

Technical University of Split, Split, Croatia

N. Godinovic, K. Lelas, R. Plestina, D. Polic, I. Puljak

University of Split, Split, Croatia

Z. Antunovic, M. Dzelalija

Institute Rudjer Boskovic, Zagreb, Croatia

V. Brigljevic, S. Duric, K. Kadija, S. Morovic

University of Cyprus, Nicosia, Cyprus

R. Fereos, M. Galanti, J. Mousa, A. Papadakis, F. Ptochos, P.A. Razis, D. Tsiakkouri, Z. Zinonos

National Institute of Chemical Physics and Biophysics, Tallinn, Estonia

A. Hektor, M. Kadastik, K. Kannike, M. Müntel, M. Raidal, L. Rebane

Helsinki Institute of Physics, Helsinki, Finland

E. Anttila, S. Czellar, J. Härkönen, A. Heikkinen, V. Karimäki, R. Kinnunen, J. Klem, M.J. Kortelainen, T. Lampén, K. Lassila-Perini, S. Lehti, T. Lindén, P. Luukka, T. Mäenpää, J. Nysten, E. Tuominen, J. Tuominiemi, D. Ungaro, L. Wendland

Lappeenranta University of Technology, Lappeenranta, Finland

K. Banzuzi, A. Korpela, T. Tuuva

Laboratoire d'Annecy-le-Vieux de Physique des Particules, IN2P3-CNRS, Annecy-le-Vieux, France

P. Nedelec, D. Sillou

DSM/IRFU, CEA/Saclay, Gif-sur-Yvette, France

M. Besancon, R. Chipaux, M. Dejardin, D. Denegri, J. Descamps, B. Fabbro, J.L. Faure, F. Ferri, S. Ganjour, F.X. Gentit, A. Givernaud, P. Gras, G. Hamel de Monchenault, P. Jarry, M.C. Lemaire, E. Locci, J. Malcles, M. Marionneau, L. Millischer, J. Rander, A. Rosowsky, D. Rousseau, M. Titov, P. Verrecchia

Laboratoire Leprince-Ringuet, Ecole Polytechnique, IN2P3-CNRS, Palaiseau, France

S. Baffioni, L. Bianchini, M. Bluj ${ }^{3}$, P. Busson, C. Charlot, L. Dobrzynski, R. Granier de Cassagnac, M. Haguenauer, P. Miné, P. Paganini, Y. Sirois, C. Thiebaux, A. Zabi 
Institut Pluridisciplinaire Hubert Curien, Université de Strasbourg, Université de Haute Alsace Mulhouse, CNRS/IN2P3, Strasbourg, France

J.-L. Agram ${ }^{4}$, A. Besson, D. Bloch, D. Bodin, J.-M. Brom, E. Conte ${ }^{4}$, F. Drouhin ${ }^{4}$, J.-C. Fontaine ${ }^{4}$, D. Gelé, U. Goerlach, L. Gross, P. Juillot, A.-C. Le Bihan, Y. Patois, J. Speck, P. Van Hove

Université de Lyon, Université Claude Bernard Lyon 1, CNRS-IN2P3, Institut de Physique Nucléaire de Lyon, Villeurbanne, France

C. Baty, M. Bedjidian, J. Blaha, G. Boudoul, H. Brun, N. Chanon, R. Chierici, D. Contardo, P. Depasse, T. Dupasquier, H. El Mamouni, F. Fassi ${ }^{5}$, J. Fay, S. Gascon, B. Ille, T. Kurca, T. Le Grand, M. Lethuillier, N. Lumb, L. Mirabito, S. Perries, M. Vander Donckt, P. Verdier

E. Andronikashvili Institute of Physics, Academy of Science, Tbilisi, Georgia

N. Djaoshvili, N. Roinishvili, V. Roinishvili

Institute of High Energy Physics and Informatization, Tbilisi State University, Tbilisi, Georgia

N. Amaglobeli

RWTH Aachen University, I. Physikalisches Institut, Aachen, Germany

R. Adolphi, G. Anagnostou, R. Brauer, W. Braunschweig, M. Edelhoff, H. Esser, L. Feld, W. Karpinski, A. Khomich, K. Klein, N. Mohr, A. Ostaptchouk, D. Pandoulas, G. Pierschel, F. Raupach, S. Schael, A. Schultz von Dratzig, G. Schwering, D. Sprenger, M. Thomas, M. Weber, B. Wittmer, M. Wlochal

RWTH Aachen University, III. Physikalisches Institut A, Aachen, Germany

O. Actis, G. Altenhöfer, W. Bender, P. Biallass, M. Erdmann, G. Fetchenhauer ${ }^{1}$, J. Frangenheim,

T. Hebbeker, G. Hilgers, A. Hinzmann, K. Hoepfner, C. Hof, M. Kirsch, T. Klimkovich,

P. Kreuzer ${ }^{1}$, D. Lanske ${ }^{\dagger}$, M. Merschmeyer, A. Meyer, B. Philipps, H. Pieta, H. Reithler, S.A. Schmitz, L. Sonnenschein, M. Sowa, J. Steggemann, H. Szczesny, D. Teyssier, C. Zeidler

RWTH Aachen University, III. Physikalisches Institut B, Aachen, Germany

M. Bontenackels, M. Davids, M. Duda, G. Flügge, H. Geenen, M. Giffels, W. Haj Ahmad, T. Hermanns, D. Heydhausen, S. Kalinin, T. Kress, A. Linn, A. Nowack, L. Perchalla, M. Poettgens, O. Pooth, P. Sauerland, A. Stahl, D. Tornier, M.H. Zoeller

Deutsches Elektronen-Synchrotron, Hamburg, Germany

M. Aldaya Martin, U. Behrens, K. Borras, A. Campbell, E. Castro, D. Dammann, G. Eckerlin, A. Flossdorf, G. Flucke, A. Geiser, D. Hatton, J. Hauk, H. Jung, M. Kasemann, I. Katkov, C. Kleinwort, H. Kluge, A. Knutsson, E. Kuznetsova, W. Lange, W. Lohmann, R. Mankel ${ }^{1}$, M. Marienfeld, A.B. Meyer, S. Miglioranzi, J. Mnich, M. Ohlerich, J. Olzem, A. Parenti, C. Rosemann, R. Schmidt, T. Schoerner-Sadenius, D. Volyanskyy, C. Wissing, W.D. Zeuner ${ }^{1}$

University of Hamburg, Hamburg, Germany

C. Autermann, F. Bechtel, J. Draeger, D. Eckstein, U. Gebbert, K. Kaschube, G. Kaussen, R. Klanner, B. Mura, S. Naumann-Emme, F. Nowak, U. Pein, C. Sander, P. Schleper, T. Schum, H. Stadie, G. Steinbrück, J. Thomsen, R. Wolf

Institut für Experimentelle Kernphysik, Karlsruhe, Germany

J. Bauer, P. Blüm, V. Buege, A. Cakir, T. Chwalek, W. De Boer, A. Dierlamm, G. Dirkes, M. Feindt, U. Felzmann, M. Frey, A. Furgeri, J. Gruschke, C. Hackstein, F. Hartmann ${ }^{1}$, S. Heier, M. Heinrich, H. Held, D. Hirschbuehl, K.H. Hoffmann, S. Honc, C. Jung, T. Kuhr, T. Liamsuwan, D. Martschei, S. Mueller, Th. Müller, M.B. Neuland, M. Niegel, O. Oberst, A. Oehler, J. Ott, T. Peiffer, D. Piparo, G. Quast, K. Rabbertz, F. Ratnikov, N. Ratnikova, M. Renz, C. Saout ${ }^{1}$, G. Sartisohn, A. Scheurer, P. Schieferdecker, F.-P. Schilling, G. Schott, H.J. Simonis, 
F.M. Stober, P. Sturm, D. Troendle, A. Trunov, W. Wagner, J. Wagner-Kuhr, M. Zeise, V. Zhukov ${ }^{6}$, E.B. Ziebarth

Institute of Nuclear Physics "Demokritos", Aghia Paraskevi, Greece

G. Daskalakis, T. Geralis, K. Karafasoulis, A. Kyriakis, D. Loukas, A. Markou, C. Markou, C. Mavrommatis, E. Petrakou, A. Zachariadou

University of Athens, Athens, Greece

L. Gouskos, P. Katsas, A. Panagiotou ${ }^{1}$

University of Ioánnina, Ioánnina, Greece

I. Evangelou, P. Kokkas, N. Manthos, I. Papadopoulos, V. Patras, F.A. Triantis

KFKI Research Institute for Particle and Nuclear Physics, Budapest, Hungary

G. Bencze ${ }^{1}$, L. Boldizsar, G. Debreczeni, C. Hajdu ${ }^{1}$, S. Hernath, P. Hidas, D. Horvath ${ }^{7}$, K. Krajczar, A. Laszlo, G. Patay, F. Sikler, N. Toth, G. Vesztergombi

Institute of Nuclear Research ATOMKI, Debrecen, Hungary

N. Beni, G. Christian, J. Imrek, J. Molnar, D. Novak, J. Palinkas, G. Szekely, Z. Szillasi ${ }^{1}$, K. Tokesi, V. Veszpremi

University of Debrecen, Debrecen, Hungary

A. Kapusi, G. Marian, P. Raics, Z. Szabo, Z.L. Trocsanyi, B. Ujvari, G. Zilizi

Panjab University, Chandigarh, India

S. Bansal, H.S. Bawa, S.B. Beri, V. Bhatnagar, M. Jindal, M. Kaur, R. Kaur, J.M. Kohli, M.Z. Mehta, N. Nishu, L.K. Saini, A. Sharma, A. Singh, J.B. Singh, S.P. Singh

University of Delhi, Delhi, India

S. Ahuja, S. Arora, S. Bhattacharya ${ }^{8}$, S. Chauhan, B.C. Choudhary, P. Gupta, S. Jain, S. Jain, M. Jha, A. Kumar, K. Ranjan, R.K. Shivpuri, A.K. Srivastava

Bhabha Atomic Research Centre, Mumbai, India

R.K. Choudhury, D. Dutta, S. Kailas, S.K. Kataria, A.K. Mohanty, L.M. Pant, P. Shukla, A. Topkar

Tata Institute of Fundamental Research - EHEP, Mumbai, India

T. Aziz, M. Guchait ${ }^{9}$, A. Gurtu, M. Maity ${ }^{10}$, D. Majumder, G. Majumder, K. Mazumdar, A. Nayak, A. Saha, K. Sudhakar

Tata Institute of Fundamental Research - HECR, Mumbai, India

S. Banerjee, S. Dugad, N.K. Mondal

Institute for Studies in Theoretical Physics \& Mathematics (IPM), Tehran, Iran

H. Arfaei, H. Bakhshiansohi, A. Fahim, A. Jafari, M. Mohammadi Najafabadi, A. Moshaii,

S. Paktinat Mehdiabadi, S. Rouhani, B. Safarzadeh, M. Zeinali

\section{University College Dublin, Dublin, Ireland}

M. Felcini

INFN Sezione di Bari ${ }^{a}$, Università di Bari ${ }^{b}$, Politecnico di Bari ${ }^{c}$, Bari, Italy

M. Abbrescia ${ }^{a, b}$, L. Barbone ${ }^{a}$, F. Chiumarulo ${ }^{a}$, A. Clemente ${ }^{a}$, A. Colaleo ${ }^{a}$, D. Creanza ${ }^{a, c}$, G. Cuscela ${ }^{a}$, N. De Filippis ${ }^{a}$, M. De Palma ${ }^{a}, b$, G. De Robertis ${ }^{a}$, G. Donvito ${ }^{a}$, F. Fedele ${ }^{a}$, L. Fiore ${ }^{a}$, M. Franco ${ }^{a}$, G. Iaselli ${ }^{a, c}$, N. Lacalamita ${ }^{a}$, F. Loddo ${ }^{a}$, L. Lusito ${ }^{a, b}$, G. Maggi ${ }^{a, c}$, M. Maggi ${ }^{a}$, N. Manna ${ }^{a, b}$, B. Marangelli ${ }^{a, b}$, S. My ${ }^{a, c}$, S. Natali ${ }^{a, b}$, S. Nuzzo ${ }^{a, b}$, G. Papagni ${ }^{a}$, S. Piccolomo ${ }^{a}$, G.A. Pierro ${ }^{a}$, C. Pinto ${ }^{a}$, A. Pompili ${ }^{a, b}$, G. Pugliese ${ }^{a, c}$, R. Rajan ${ }^{a}$, A. Ranieri ${ }^{a}$, F. Romano ${ }^{a, c}$, 
G. Roselli ${ }^{a, b}$, G. Selvaggi ${ }^{a, b}$, Y. Shinde ${ }^{a}$, L. Silvestris $^{a}$, S. Tupputi ${ }^{a, b}$, G. Zito $^{a}$

INFN Sezione di Bologna ${ }^{a}$, Universita di Bologna ${ }^{b}$, Bologna, Italy

G. Abbiendi ${ }^{a}$, W. Bacchi ${ }^{a, b}$, A.C. Benvenuti ${ }^{a}$, M. Boldini ${ }^{a}$, D. Bonacorsi ${ }^{a}$, S. BraibantGiacomelli $^{a, b}$, V.D. Cafaro ${ }^{a}$, S.S. Caiazza ${ }^{a}$, P. Capiluppi ${ }^{a, b}$, A. Castro ${ }^{a, b}$, F.R. Cavallo ${ }^{a}$, G. Codispoti ${ }^{a, b}$, M. Cuffiani ${ }^{a, b}$, I. D'Antone ${ }^{a}$, G.M. Dallavalle ${ }^{a, 1}$, F. Fabbri ${ }^{a}$, A. Fanfani ${ }^{a, b}$, D. Fasanella ${ }^{a}$, P. Giacomelli ${ }^{a}$, V. Giordano ${ }^{a}$, M. Giunta ${ }^{a}, 1$, C. Grandi ${ }^{a}$, M. Guerzoni ${ }^{a}$, S. Marcellini ${ }^{a}$, G. Masetti ${ }^{a}, b$, A. Montanari ${ }^{a}$, F.L. Navarria ${ }^{a}, b$, F. Odorici ${ }^{a}$, G. Pellegrini ${ }^{a}$, A. Perrotta ${ }^{a}$, A.M. Rossi ${ }^{a, b}$, T. Rovelli ${ }^{a, b}$, G. Siroli ${ }^{a}, b$, G. Torromeo ${ }^{a}$, R. Travaglini ${ }^{a, b}$

INFN Sezione di Catania ${ }^{a}$, Universita di Catania ${ }^{b}$, Catania, Italy

S. Albergo ${ }^{a, b}$, S. Costa ${ }^{a, b}$, R. Potenza ${ }^{a, b}$, A. Tricomi ${ }^{a, b}$, C. Tuve ${ }^{a}$

INFN Sezione di Firenze ${ }^{a}$, Universita di Firenze ${ }^{b}$, Firenze, Italy

G. Barbagli ${ }^{a}$, G. Broccolo ${ }^{a, b}$, V. Ciullii ${ }^{a}, b$, C. Civinini ${ }^{a}$, R. D'Alessandro ${ }^{a, b}$, E. Focardi ${ }^{a, b}$, S. Frosali ${ }^{a, b}$, E. Gallo ${ }^{a}$, C. Genta ${ }^{a, b}$, G. Landi ${ }^{a, b}$, P. Lenzi ${ }^{a, b, 1}$, M. Meschini ${ }^{a}$, S. Paoletti ${ }^{a}$, G. Sguazzoni ${ }^{a}$, A. Tropiano ${ }^{a}$

INFN Laboratori Nazionali di Frascati, Frascati, Italy

L. Benussi, M. Bertani, S. Bianco, S. Colafranceschi ${ }^{11}$, D. Colonna ${ }^{11}$, F. Fabbri, M. Giardoni, L. Passamonti, D. Piccolo, D. Pierluigi, B. Ponzio, A. Russo

INFN Sezione di Genova, Genova, Italy

P. Fabbricatore, R. Musenich

INFN Sezione di Milano-Biccoca ${ }^{a}$, Universita di Milano-Bicocca ${ }^{b}$, Milano, Italy

A. Benaglia ${ }^{a}$, M. Calloni ${ }^{a}$, G.B. Cerati ${ }^{a}, b, 1$, P. D $^{\prime}$ Angelo $^{a}$, F. De Guio ${ }^{a}$, F.M. Farina ${ }^{a}$, A. Ghezzi $^{a}$, P. Govoni ${ }^{a}, b$, M. Malberti ${ }^{a}, b, 1$, S. Malvezzi ${ }^{a}$, A. Martelli ${ }^{a}$, D. Menasce ${ }^{a}$, V. Miccio ${ }^{a}, b$, L. Moroni $^{a}$, P. Negri ${ }^{a, b}$, M. Paganoni ${ }^{a, b}$, D. Pedrini ${ }^{a}$, A. Pullia ${ }^{a, b}$, S. Ragazzi ${ }^{a}, b$, N. Redaelli ${ }^{a}$, S. Sala ${ }^{a}$, R. Salerno ${ }^{a, b}$, T. Tabarelli de Fatis ${ }^{a, b}$, V. Tancini ${ }^{a, b}$, S. Taroni ${ }^{a, b}$

INFN Sezione di Napoli ${ }^{a}$, Universita di Napoli "Federico II" $b$, Napoli, Italy

S. Buontempo ${ }^{a}$, N. Cavallo ${ }^{a}$, A. Cimmino ${ }^{a, b}, 1$, M. De Gruttola ${ }^{a, b, 1}$, F. Fabozzi $^{a, 12}$, A.O.M. Iorio ${ }^{a}$, L. Lista ${ }^{a}$, D. Lomidze ${ }^{a}$, P. Noli ${ }^{a, b}$, P. Paolucci ${ }^{a}$, C. Sciacca ${ }^{a, b}$

INFN Sezione di Padova ${ }^{a}$, Università di Padova ${ }^{b}$, Padova, Italy

P. Azzi ${ }^{a, 1}$, N. Bacchetta ${ }^{a}$, L. Barcellan ${ }^{a}$, P. Bellan $^{a, b, 1}$, M. Bellato ${ }^{a}$, M. Benettoni ${ }^{a}$, M. Biasotto $^{a, 13}$, D. Bisello ${ }^{a, b}$, E. Borsato ${ }^{a, b}$, A. Branca ${ }^{a}$, R. Carlin ${ }^{a}, b$, L. Castellani ${ }^{a}$, P. Checchia ${ }^{a}$, E. Conti ${ }^{a}$, F. Dal Corso ${ }^{a}$, M. De Mattia ${ }^{a, b}$, T. Dorigo ${ }^{a}$, U. Dosselli ${ }^{a}$, F. Fanzago ${ }^{a}$, F. Gasparini ${ }^{a, b}$, U. Gasparini ${ }^{a, b}$, P. Giubilato ${ }^{a, b}$, F. Gonella ${ }^{a}$, A. Gresele ${ }^{a, 14}$, M. Gulmini ${ }^{a, 13}$, A. Kaminskiy ${ }^{a, b}$, S. Lacaprara ${ }^{a, 13}$, I. Lazzizzera ${ }^{a, 14}$, M. Margoni ${ }^{a, b}$, G. Maron $^{a, 13}$, S. Mattiazzo ${ }^{a, b}$, M. Mazzucato ${ }^{a}$, M. Meneghelli ${ }^{a}$, A.T. Meneguzzo ${ }^{a, b}$, M. Michelotto ${ }^{a}$, F. Montecassiano ${ }^{a}$, M. Nespolo ${ }^{a}$, M. Passaseo ${ }^{a}$, M. Pegoraro ${ }^{a}$, L. Perrozzi ${ }^{a}$, N. Pozzobon ${ }^{a, b}$, P. Ronchese ${ }^{a, b}$, F. Simonetto ${ }^{a, b}$, N. Toniolo ${ }^{a}$, E. Torassa ${ }^{a}$, M. Tosi ${ }^{a, b}$, A. Triossi ${ }^{a}$, S. Vanini ${ }^{a, b}$, S. Ventura ${ }^{a}$, P. Zotto ${ }^{a, b}$, G. Zumerle ${ }^{a, b}$

INFN Sezione di Pavia ${ }^{a}$, Universita di Pavia ${ }^{b}$, Pavia, Italy

P. Baesso ${ }^{a, b}$, U. Berzano ${ }^{a}$, S. Bricola ${ }^{a}$, M.M. Necchi ${ }^{a, b}$, D. Pagano ${ }^{a, b}$, S.P. Ratti ${ }^{a, b}$, C. Riccardi ${ }^{a, b}$, P. Torre ${ }^{a, b}$, A. Vicini ${ }^{a}$, P. Vitulo ${ }^{a, b}$, C. Viviani ${ }^{a, b}$

INFN Sezione di Perugia ${ }^{a}$, Universita di Perugia ${ }^{b}$, Perugia, Italy

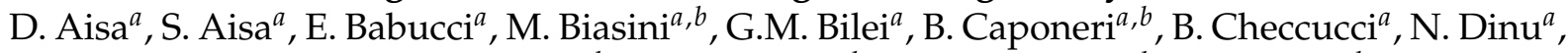

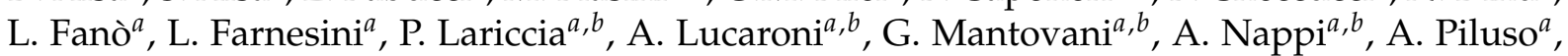
V. Postolache ${ }^{a}$, A. Santocchia ${ }^{a, b}$, L. Servoli $^{a}$, D. Tonoiu ${ }^{a}$, A. Vedaee ${ }^{a}$, R. Volpe $^{a, b}$ 
INFN Sezione di Pisa ${ }^{a}$, Universita di Pisa ${ }^{b}$, Scuola Normale Superiore di Pisa ${ }^{c}$, Pisa, Italy P. Azzurri ${ }^{a, c}$, G. Bagliesi ${ }^{a}$, J. Bernardini ${ }^{a, b}$, L. Berretta ${ }^{a}$, T. Boccali ${ }^{a}$, A. Bocci ${ }^{a, c}$, L. Borrello ${ }^{a, c}$, F. Bosi ${ }^{a}$, F. Calzolari ${ }^{a}$, R. Castaldi ${ }^{a}$, R. Dell'Orso ${ }^{a}$, F. Fiori ${ }^{a}, b$, L. Foà ${ }^{a, c}$, S. Gennai ${ }^{a, c}$, A. Giassi ${ }^{a}$, A. Kraan ${ }^{a}$, F. Ligabue ${ }^{a, c}$, T. Lomtadze ${ }^{a}$, F. Mariani ${ }^{a}$, L. Martini ${ }^{a}$, M. Massa ${ }^{a}$, A. Messineo ${ }^{a}, b$, A. Moggi ${ }^{a}$, F. Palla ${ }^{a}$, F. Palmonari ${ }^{a}$, G. Petragnani ${ }^{a}$, G. Petrucciani ${ }^{a}, c$, F. Raffaelli ${ }^{a}$, S. Sarkar ${ }^{a}$, G. Segneri ${ }^{a}$, A.T. Serban ${ }^{a}$, P. Spagnolo ${ }^{a, 1}$, R. Tenchini ${ }^{a, 1}$, S. Tolaini ${ }^{a}$, G. Tonellia ${ }^{a, b}, 1$, A. Venturi ${ }^{a}$, P.G. Verdini ${ }^{a}$

INFN Sezione di Roma ${ }^{a}$, Universita di Roma "La Sapienza" ${ }^{b}$, Roma, Italy

S. Baccaro ${ }^{a, 15}$, L. Barone ${ }^{a, b}$, A. Bartoloni ${ }^{a}$, F. Cavallari ${ }^{a, 1}$, I. Dafinei ${ }^{a}$, D. Del Re ${ }^{a, b}$, E. Di Marco $^{a, b}$, M. Diemoz ${ }^{a}$, D. Franci ${ }^{a, b}$, E. Longo ${ }^{a, b}$, G. Organtini ${ }^{a, b}$, A. Palma ${ }^{a, b}$, F. Pandolfi ${ }^{a, b}$, R. Paramatti ${ }^{a, 1}$, F. Pellegrino ${ }^{a}$, S. Rahatlou ${ }^{a, b}$, C. Rovelli $^{a}$

INFN Sezione di Torino ${ }^{a}$, Università di Torino ${ }^{b}$, Università del Piemonte Orientale (Novara) ${ }^{c}$, Torino, Italy

G. Alampi ${ }^{a}$, N. Amapane ${ }^{a, b}$, R. Arcidiacono ${ }^{a, b}$, S. Argiro ${ }^{a, b}$, M. Arneodo ${ }^{a, c}$, C. Biino ${ }^{a}$, M.A. Borgia ${ }^{a, b}$, C. Botta ${ }^{a, b}$, N. Cartiglia ${ }^{a}$, R. Castello ${ }^{a, b}$, G. Cerminara ${ }^{a, b}$, M. Costa ${ }^{a, b}$, D. Dattola ${ }^{a}$, G. Dellacasa ${ }^{a}$, N. Demaria ${ }^{a}$, G. Dughera ${ }^{a}$, F. Dumitrache ${ }^{a}$, A. Graziano ${ }^{a, b}$, C. Mariotti ${ }^{a}$, M. Marone ${ }^{a, b}$, S. Masellii ${ }^{a}$, E. Migliore ${ }^{a, b}$, G. Mila ${ }^{a, b}$, V. Monaco ${ }^{a, b}$, M. Musich $^{a, b}$, M. Nervo ${ }^{a, b}$, M.M. Obertino ${ }^{a, c}$, S. Oggero ${ }^{a, b}$, R. Panero ${ }^{a}$, N. Pastrone ${ }^{a}$, M. Pelliccioni ${ }^{a, b}$, A. Romero ${ }^{a, b}$, M. Ruspa ${ }^{a, c}$, R. Sacchi ${ }^{a, b}$, A. Solano ${ }^{a, b}$, A. Staiano ${ }^{a}$, P.P. Trapani ${ }^{a, b, 1}$, D. Trocino ${ }^{a, b}$, A. Vilela Pereira ${ }^{a, b}$, L. Visca $^{a, b}$, A. Zampieri ${ }^{a}$

INFN Sezione di Trieste ${ }^{a}$, Universita di Trieste ${ }^{b}$, Trieste, Italy

F. Ambroglini ${ }^{a, b}$, S. Belforte ${ }^{a}$, F. Cossutti ${ }^{a}$, G. Della Ricca ${ }^{a, b}$, B. Gobbo ${ }^{a}$, A. Penzo $^{a}$

Kyungpook National University, Daegu, Korea

S. Chang, J. Chung, D.H. Kim, G.N. Kim, D.J. Kong, H. Park, D.C. Son

Wonkwang University, Iksan, Korea

S.Y. Bahk

Chonnam National University, Kwangju, Korea

S. Song

Konkuk University, Seoul, Korea

S.Y. Jung

Korea University, Seoul, Korea

B. Hong, H. Kim, J.H. Kim, K.S. Lee, D.H. Moon, S.K. Park, H.B. Rhee, K.S. Sim

\section{Seoul National University, Seoul, Korea}

J. Kim

University of Seoul, Seoul, Korea

M. Choi, G. Hahn, I.C. Park

Sungkyunkwan University, Suwon, Korea

S. Choi, Y. Choi, J. Goh, H. Jeong, T.J. Kim, J. Lee, S. Lee

Vilnius University, Vilnius, Lithuania

M. Janulis, D. Martisiute, P. Petrov, T. Sabonis

Centro de Investigacion y de Estudios Avanzados del IPN, Mexico City, Mexico

H. Castilla Valdez ${ }^{1}$, A. Sánchez Hernández 
Universidad Iberoamericana, Mexico City, Mexico

S. Carrillo Moreno

Universidad Autónoma de San Luis Potosí, San Luis Potosí, Mexico

A. Morelos Pineda

University of Auckland, Auckland, New Zealand

P. Allfrey, R.N.C. Gray, D. Krofcheck

University of Canterbury, Christchurch, New Zealand

N. Bernardino Rodrigues, P.H. Butler, T. Signal, J.C. Williams

National Centre for Physics, Quaid-I-Azam University, Islamabad, Pakistan

M. Ahmad, I. Ahmed, W. Ahmed, M.I. Asghar, M.I.M. Awan, H.R. Hoorani, I. Hussain, W.A. Khan, T. Khurshid, S. Muhammad, S. Qazi, H. Shahzad

\section{Institute of Experimental Physics, Warsaw, Poland}

M. Cwiok, R. Dabrowski, W. Dominik, K. Doroba, M. Konecki, J. Krolikowski, K. Pozniak ${ }^{16}$, R. Romaniuk, W. Zabolotny ${ }^{16}$, P. Zych

\section{Soltan Institute for Nuclear Studies, Warsaw, Poland}

T. Frueboes, R. Gokieli, L. Goscilo, M. Górski, M. Kazana, K. Nawrocki, M. Szleper, G. Wrochna, P. Zalewski

\section{Laboratório de Instrumentação e Física Experimental de Partículas, Lisboa, Portugal}

N. Almeida, L. Antunes Pedro, P. Bargassa, A. David, P. Faccioli, P.G. Ferreira Parracho, M. Freitas Ferreira, M. Gallinaro, M. Guerra Jordao, P. Martins, G. Mini, P. Musella, J. Pela, L. Raposo, P.Q. Ribeiro, S. Sampaio, J. Seixas, J. Silva, P. Silva, D. Soares, M. Sousa, J. Varela, H.K. Wöhri

\section{Joint Institute for Nuclear Research, Dubna, Russia}

I. Altsybeev, I. Belotelov, P. Bunin, Y. Ershov, I. Filozova, M. Finger, M. Finger Jr., A. Golunov, I. Golutvin, N. Gorbounov, V. Kalagin, A. Kamenev, V. Karjavin, V. Konoplyanikov, V. Korenkov, G. Kozlov, A. Kurenkov, A. Lanev, A. Makankin, V.V. Mitsyn, P. Moisenz, E. Nikonov, D. Oleynik, V. Palichik, V. Perelygin, A. Petrosyan, R. Semenov, S. Shmatov, V. Smirnov, D. Smolin, E. Tikhonenko, S. Vasil'ev, A. Vishnevskiy, A. Volodko, A. Zarubin, V. Zhiltsov

\section{Petersburg Nuclear Physics Institute, Gatchina (St Petersburg), Russia}

N. Bondar, L. Chtchipounov, A. Denisov, Y. Gavrikov, G. Gavrilov, V. Golovtsov, Y. Ivanov, V. Kim, V. Kozlov, P. Levchenko, G. Obrant, E. Orishchin, A. Petrunin, Y. Shcheglov, A. Shchetkovskiy, V. Sknar, I. Smirnov, V. Sulimov, V. Tarakanov, L. Uvarov, S. Vavilov, G. Velichko, S. Volkov, A. Vorobyev

\section{Institute for Nuclear Research, Moscow, Russia}

Yu. Andreev, A. Anisimov, P. Antipov, A. Dermenev, S. Gninenko, N. Golubev, M. Kirsanov, N. Krasnikov, V. Matveev, A. Pashenkov, V.E. Postoev, A. Solovey, A. Solovey, A. Toropin, S. Troitsky

\section{Institute for Theoretical and Experimental Physics, Moscow, Russia}

A. Baud, V. Epshteyn, V. Gavrilov, N. Ilina, V. Kaftanov ${ }^{\dagger}$, V. Kolosov, M. Kossov' ${ }^{1}$, A. Krokhotin, S. Kuleshov, A. Oulianov, G. Safronov, S. Semenov, I. Shreyber, V. Stolin, E. Vlasov, A. Zhokin

Moscow State University, Moscow, Russia

E. Boos, M. Dubinin ${ }^{17}$, L. Dudko, A. Ershov, A. Gribushin, V. Klyukhin, O. Kodolova, I. Lokhtin,

S. Petrushanko, L. Sarycheva, V. Savrin, A. Snigirev, I. Vardanyan 


\section{P.N. Lebedev Physical Institute, Moscow, Russia}

I. Dremin, M. Kirakosyan, N. Konovalova, S.V. Rusakov, A. Vinogradov

State Research Center of Russian Federation, Institute for High Energy Physics, Protvino, Russia

S. Akimenko, A. Artamonov, I. Azhgirey, S. Bitioukov, V. Burtovoy, V. Grishin ${ }^{1}$, V. Kachanov, D. Konstantinov, V. Krychkine, A. Levine, I. Lobov, V. Lukanin, Y. Mel'nik, V. Petrov, R. Ryutin, S. Slabospitsky, A. Sobol, A. Sytine, L. Tourtchanovitch, S. Troshin, N. Tyurin, A. Uzunian, A. Volkov

Vinca Institute of Nuclear Sciences, Belgrade, Serbia

P. Adzic, M. Djordjevic, D. Jovanovic ${ }^{18}$, D. Krpic ${ }^{18}$, D. Maletic, J. Puzovic ${ }^{18}$, N. Smiljkovic

Centro de Investigaciones Energéticas Medioambientales y Tecnológicas (CIEMAT), Madrid, Spain

M. Aguilar-Benitez, J. Alberdi, J. Alcaraz Maestre, P. Arce, J.M. Barcala, C. Battilana, C. Burgos Lazaro, J. Caballero Bejar, E. Calvo, M. Cardenas Montes, M. Cepeda, M. Cerrada, M. Chamizo Llatas, F. Clemente, N. Colino, M. Daniel, B. De La Cruz, A. Delgado Peris, C. Diez Pardos, C. Fernandez Bedoya, J.P. Fernández Ramos, A. Ferrando, J. Flix, M.C. Fouz, P. Garcia-Abia, A.C. Garcia-Bonilla, O. Gonzalez Lopez, S. Goy Lopez, J.M. Hernandez, M.I. Josa, J. Marin, G. Merino, J. Molina, A. Molinero, J.J. Navarrete, J.C. Oller, J. Puerta Pelayo, L. Romero, J. Santaolalla, C. Villanueva Munoz, C. Willmott, C. Yuste

Universidad Autónoma de Madrid, Madrid, Spain

C. Albajar, M. Blanco Otano, J.F. de Trocóniz, A. Garcia Raboso, J.O. Lopez Berengueres

Universidad de Oviedo, Oviedo, Spain

J. Cuevas, J. Fernandez Menendez, I. Gonzalez Caballero, L. Lloret Iglesias, H. Naves Sordo, J.M. Vizan Garcia

Instituto de Física de Cantabria (IFCA), CSIC-Universidad de Cantabria, Santander, Spain I.J. Cabrillo, A. Calderon, S.H. Chuang, I. Diaz Merino, C. Diez Gonzalez, J. Duarte Campderros, M. Fernandez, G. Gomez, J. Gonzalez Sanchez, R. Gonzalez Suarez, C. Jorda, P. Lobelle Pardo, A. Lopez Virto, J. Marco, R. Marco, C. Martinez Rivero, P. Martinez Ruiz del Arbol, F. Matorras, T. Rodrigo, A. Ruiz Jimeno, L. Scodellaro, M. Sobron Sanudo, I. Vila, R. Vilar Cortabitarte

\section{CERN, European Organization for Nuclear Research, Geneva, Switzerland}

D. Abbaneo, E. Albert, M. Alidra, S. Ashby, E. Auffray, J. Baechler, P. Baillon, A.H. Ball, S.L. Bally, D. Barney, F. Beaudette ${ }^{19}$, R. Bellan, D. Benedetti, G. Benelli, C. Bernet, P. Bloch, S. Bolognesi, M. Bona, J. Bos, N. Bourgeois, T. Bourrel, H. Breuker, K. Bunkowski, D. Campi, T. Camporesi, E. Cano, A. Cattai, J.P. Chatelain, M. Chauvey, T. Christiansen, J.A. Coarasa Perez, A. Conde Garcia, R. Covarelli, B. Curé, A. De Roeck, V. Delachenal, D. Deyrail, S. Di Vincenzo $^{20}$, S. Dos Santos, T. Dupont, L.M. Edera, A. Elliott-Peisert, M. Eppard, M. Favre, N. Frank, W. Funk, A. Gaddi, M. Gastal, M. Gateau, H. Gerwig, D. Gigi, K. Gill, D. Giordano, J.P. Girod, F. Glege, R. Gomez-Reino Garrido, R. Goudard, S. Gowdy, R. Guida, L. Guiducci, J. Gutleber, M. Hansen, C. Hartl, J. Harvey, B. Hegner, H.F. Hoffmann, A. Holzner, A. Honma, M. Huhtinen, V. Innocente, P. Janot, G. Le Godec, P. Lecoq, C. Leonidopoulos, R. Loos, C. Lourenço, A. Lyonnet, A. Macpherson, N. Magini, J.D. Maillefaud, G. Maire, T. Mäki, L. Malgeri, M. Mannelli, L. Masetti, F. Meijers, P. Meridiani, S. Mersi, E. Meschi, A. Meynet Cordonnier, R. Moser, M. Mulders, J. Mulon, M. Noy, A. Oh, G. Olesen, A. Onnela, T. Orimoto, L. Orsini, E. Perez, G. Perinic, J.F. Pernot, P. Petagna, P. Petiot, A. Petrilli, A. Pfeiffer, M. Pierini, M. Pimiä, R. Pintus, B. Pirollet, H. Postema, A. Racz, S. Ravat, S.B. Rew, J. Rodrigues Antunes, 
G. Rolandi ${ }^{21}$, M. Rovere, V. Ryjov, H. Sakulin, D. Samyn, H. Sauce, C. Schäfer, W.D. Schlatter, M. Schröder, C. Schwick, A. Sciaba, I. Segoni, A. Sharma, N. Siegrist, P. Siegrist, N. Sinanis, T. Sobrier, P. Sphicas ${ }^{22}$, D. Spiga, M. Spiropulu ${ }^{17}$, F. Stöckli, P. Traczyk, P. Tropea, J. Troska, A. Tsirou, L. Veillet, G.I. Veres, M. Voutilainen, P. Wertelaers, M. Zanetti

Paul Scherrer Institut, Villigen, Switzerland

W. Bertl, K. Deiters, W. Erdmann, K. Gabathuler, R. Horisberger, Q. Ingram, H.C. Kaestli, S. König, D. Kotlinski, U. Langenegger, F. Meier, D. Renker, T. Rohe, J. Sibille ${ }^{23}$, A. Starodumov ${ }^{24}$

Institute for Particle Physics, ETH Zurich, Zurich, Switzerland

B. Betev, L. Caminada ${ }^{25}$, Z. Chen, S. Cittolin, D.R. Da Silva Di Calafiori, S. Dambach ${ }^{25}$, G. Dissertori, M. Dittmar, C. Eggel ${ }^{25}$, J. Eugster, G. Faber, K. Freudenreich, C. Grab, A. Hervé, W. Hintz, P. Lecomte, P.D. Luckey, W. Lustermann, C. Marchica ${ }^{25}$, P. Milenovic ${ }^{26}$, F. Moortgat, A. Nardulli, F. Nessi-Tedaldi, L. Pape, F. Pauss, T. Punz, A. Rizzi, F.J. Ronga, L. Sala, A.K. Sanchez, M.-C. Sawley, V. Sordini, B. Stieger, L. Tauscher ${ }^{\dagger}$, A. Thea, K. Theofilatos, D. Treille, P. Trüb ${ }^{25}$, M. Weber, L. Wehrli, J. Weng, S. Zelepoukine ${ }^{27}$

Universität Zürich, Zurich, Switzerland

C. Amsler, V. Chiochia, S. De Visscher, C. Regenfus, P. Robmann, T. Rommerskirchen, A. Schmidt, D. Tsirigkas, L. Wilke

National Central University, Chung-Li, Taiwan

Y.H. Chang, E.A. Chen, W.T. Chen, A. Go, C.M. Kuo, S.W. Li, W. Lin

National Taiwan University (NTU), Taipei, Taiwan

P. Bartalini, P. Chang, Y. Chao, K.F. Chen, W.-S. Hou, Y. Hsiung, Y.J. Lei, S.W. Lin, R.-S. Lu, J. Schümann, J.G. Shiu, Y.M. Tzeng, K. Ueno, Y. Velikzhanin, C.C. Wang, M. Wang

Cukurova University, Adana, Turkey

A. Adiguzel, A. Ayhan, A. Azman Gokce, M.N. Bakirci, S. Cerci, I. Dumanoglu, E. Eskut, S. Girgis, E. Gurpinar, I. Hos, T. Karaman, T. Karaman, A. Kayis Topaksu, P. Kurt, G. Önengüt, G. Önengüt Gökbulut, K. Ozdemir, S. Ozturk, A. Polatöz, K. Sogut ${ }^{28}$, B. Tali, H. Topakli, D. Uzun, L.N. Vergili, M. Vergili

Middle East Technical University, Physics Department, Ankara, Turkey

I.V. Akin, T. Aliev, S. Bilmis, M. Deniz, H. Gamsizkan, A.M. Guler, K. Öcalan, M. Serin, R. Sever, U.E. Surat, M. Zeyrek

Bogaziçi University, Department of Physics, Istanbul, Turkey

M. Deliomeroglu, D. Demir ${ }^{29}$, E. Gülmez, A. Halu, B. Isildak, M. Kaya ${ }^{30}$, O. Kaya ${ }^{30}$, S. Ozkorucuklu $^{31}$, N. Sonmez ${ }^{32}$

National Scientific Center, Kharkov Institute of Physics and Technology, Kharkov, Ukraine L. Levchuk, S. Lukyanenko, D. Soroka, S. Zub

University of Bristol, Bristol, United Kingdom

F. Bostock, J.J. Brooke, T.L. Cheng, D. Cussans, R. Frazier, J. Goldstein, N. Grant, M. Hansen, G.P. Heath, H.F. Heath, C. Hill, B. Huckvale, J. Jackson, C.K. Mackay, S. Metson, D.M. Newbold ${ }^{33}$, K. Nirunpong, V.J. Smith, J. Velthuis, R. Walton

Rutherford Appleton Laboratory, Didcot, United Kingdom

K.W. Bell, C. Brew, R.M. Brown, B. Camanzi, D.J.A. Cockerill, J.A. Coughlan, N.I. Geddes, K. Harder, S. Harper, B.W. Kennedy, P. Murray, C.H. Shepherd-Themistocleous, I.R. Tomalin, J.H. Williams ${ }^{\dagger}$, W.J. Womersley, S.D. Worm 
Imperial College, University of London, London, United Kingdom

R. Bainbridge, G. Ball, J. Ballin, R. Beuselinck, O. Buchmuller, D. Colling, N. Cripps, G. Davies, M. Della Negra, C. Foudas, J. Fulcher, D. Futyan, G. Hall, J. Hays, G. Iles, G. Karapostoli, B.C. MacEvoy, A.-M. Magnan, J. Marrouche, J. Nash, A. Nikitenko ${ }^{24}$, A. Papageorgiou, M. Pesaresi, K. Petridis, M. Pioppi ${ }^{34}$, D.M. Raymond, N. Rompotis, A. Rose, M.J. Ryan, C. Seez, P. Sharp, G. Sidiropoulos ${ }^{1}$, M. Stettler, M. Stoye, M. Takahashi, A. Tapper, C. Timlin, S. Tourneur, M. Vazquez Acosta, T. Virdee ${ }^{1}$, S. Wakefield, D. Wardrope, T. Whyntie, M. Wingham

\section{Brunel University, Uxbridge, United Kingdom}

J.E. Cole, I. Goitom, P.R. Hobson, A. Khan, P. Kyberd, D. Leslie, C. Munro, I.D. Reid, C. Siamitros, R. Taylor, L. Teodorescu, I. Yaselli

\section{Boston University, Boston, USA}

T. Bose, M. Carleton, E. Hazen, A.H. Heering, A. Heister, J. St. John, P. Lawson, D. Lazic, D. Osborne, J. Rohlf, L. Sulak, S. Wu

\section{Brown University, Providence, USA}

J. Andrea, A. Avetisyan, S. Bhattacharya, J.P. Chou, D. Cutts, S. Esen, G. Kukartsev, G. Landsberg, M. Narain, D. Nguyen, T. Speer, K.V. Tsang

\section{University of California, Davis, Davis, USA}

R. Breedon, M. Calderon De La Barca Sanchez, M. Case, D. Cebra, M. Chertok, J. Conway, P.T. Cox, J. Dolen, R. Erbacher, E. Friis, W. Ko, A. Kopecky, R. Lander, A. Lister, H. Liu, S. Maruyama, T. Miceli, M. Nikolic, D. Pellett, J. Robles, M. Searle, J. Smith, M. Squires, J. Stilley, M. Tripathi, R. Vasquez Sierra, C. Veelken

\section{University of California, Los Angeles, Los Angeles, USA}

V. Andreev, K. Arisaka, D. Cline, R. Cousins, S. Erhan ${ }^{1}$, J. Hauser, M. Ignatenko, C. Jarvis, J. Mumford, C. Plager, G. Rakness, P. Schlein ${ }^{\dagger}$, J. Tucker, V. Valuev, R. Wallny, X. Yang

University of California, Riverside, Riverside, USA

J. Babb, M. Bose, A. Chandra, R. Clare, J.A. Ellison, J.W. Gary, G. Hanson, G.Y. Jeng, S.C. Kao, F. Liu, H. Liu, A. Luthra, H. Nguyen, G. Pasztor ${ }^{35}$, A. Satpathy, B.C. Shen ${ }^{\dagger}$, R. Stringer, J. Sturdy, V. Sytnik, R. Wilken, S. Wimpenny

\section{University of California, San Diego, La Jolla, USA}

J.G. Branson, E. Dusinberre, D. Evans, F. Golf, R. Kelley, M. Lebourgeois, J. Letts, E. Lipeles, B. Mangano, J. Muelmenstaedt, M. Norman, S. Padhi, A. Petrucci, H. Pi, M. Pieri, R. Ranieri, M. Sani, V. Sharma, S. Simon, F. Würthwein, A. Yagil

\section{University of California, Santa Barbara, Santa Barbara, USA}

C. Campagnari, M. D’Alfonso, T. Danielson, J. Garberson, J. Incandela, C. Justus, P. Kalavase, S.A. Koay, D. Kovalskyi, V. Krutelyov, J. Lamb, S. Lowette, V. Pavlunin, F. Rebassoo, J. Ribnik, J. Richman, R. Rossin, D. Stuart, W. To, J.R. Vlimant, M. Witherell

\section{California Institute of Technology, Pasadena, USA}

A. Apresyan, A. Bornheim, J. Bunn, M. Chiorboli, M. Gataullin, D. Kcira, V. Litvine, Y. Ma, H.B. Newman, C. Rogan, V. Timciuc, J. Veverka, R. Wilkinson, Y. Yang, L. Zhang, K. Zhu, R.Y. Zhu

\section{Carnegie Mellon University, Pittsburgh, USA}

B. Akgun, R. Carroll, T. Ferguson, D.W. Jang, S.Y. Jun, M. Paulini, J. Russ, N. Terentyev, H. Vogel, I. Vorobiev 


\section{University of Colorado at Boulder, Boulder, USA}

J.P. Cumalat, M.E. Dinardo, B.R. Drell, W.T. Ford, B. Heyburn, E. Luiggi Lopez, U. Nauenberg, K. Stenson, K. Ulmer, S.R. Wagner, S.L. Zang

\section{Cornell University, Ithaca, USA}

L. Agostino, J. Alexander, F. Blekman, D. Cassel, A. Chatterjee, S. Das, L.K. Gibbons, B. Heltsley, W. Hopkins, A. Khukhunaishvili, B. Kreis, V. Kuznetsov, J.R. Patterson, D. Puigh, A. Ryd, X. Shi, S. Stroiney, W. Sun, W.D. Teo, J. Thom, J. Vaughan, Y. Weng, P. Wittich

\section{Fairfield University, Fairfield, USA}

C.P. Beetz, G. Cirino, C. Sanzeni, D. Winn

\section{Fermi National Accelerator Laboratory, Batavia, USA}

S. Abdullin, M.A. Afaq ${ }^{1}$, M. Albrow, B. Ananthan, G. Apollinari, M. Atac, W. Badgett, L. Bagby, J.A. Bakken, B. Baldin, S. Banerjee, K. Banicz, L.A.T. Bauerdick, A. Beretvas, J. Berryhill, P.C. Bhat, K. Biery, M. Binkley, I. Bloch, F. Borcherding, A.M. Brett, K. Burkett, J.N. Butler, V. Chetluru, H.W.K. Cheung, F. Chlebana, I. Churin, S. Cihangir, M. Crawford, W. Dagenhart, M. Demarteau, G. Derylo, D. Dykstra, D.P. Eartly, J.E. Elias, V.D. Elvira, D. Evans, L. Feng, M. Fischler, I. Fisk, S. Foulkes, J. Freeman, P. Gartung, E. Gottschalk, T. Grassi, D. Green, Y. Guo, O. Gutsche, A. Hahn, J. Hanlon, R.M. Harris, B. Holzman, J. Howell, D. Hufnagel, E. James, H. Jensen, M. Johnson, C.D. Jones, U. Joshi, E. Juska, J. Kaiser, B. Klima, S. Kossiakov, K. Kousouris, S. Kwan, C.M. Lei, P. Limon, J.A. Lopez Perez, S. Los, L. Lueking, G. Lukhanin, S. Lusin ${ }^{1}$, J. Lykken, K. Maeshima, J.M. Marraffino, D. Mason, P. McBride, T. Miao, K. Mishra, S. Moccia, R. Mommsen, S. Mrenna, A.S. Muhammad, C. Newman-Holmes, C. Noeding, V. O’Dell, O. Prokofyev, R. Rivera, C.H. Rivetta, A. Ronzhin, P. Rossman, S. Ryu, V. Sekhri, E. Sexton-Kennedy, I. Sfiligoi, S. Sharma, T.M. Shaw, D. Shpakov, E. Skup, R.P. Smith ${ }^{\dagger}$, A. Soha, W.J. Spalding, L. Spiegel, I. Suzuki, P. Tan, W. Tanenbaum, S. Tkaczyk ${ }^{1}$, R. Trentadue ${ }^{1}$, L. Uplegger, E.W. Vaandering, R. Vidal, J. Whitmore, E. Wicklund, W. Wu, J. Yarba, F. Yumiceva, J.C. Yun

\section{University of Florida, Gainesville, USA}

D. Acosta, P. Avery, V. Barashko, D. Bourilkov, M. Chen, G.P. Di Giovanni, D. Dobur, A. Drozdetskiy, R.D. Field, Y. Fu, I.K. Furic, J. Gartner, D. Holmes, B. Kim, S. Klimenko, J. Konigsberg, A. Korytov, K. Kotov, A. Kropivnitskaya, T. Kypreos, A. Madorsky, K. Matchev, G. Mitselmakher, Y. Pakhotin, J. Piedra Gomez, C. Prescott, V. Rapsevicius, R. Remington, M. Schmitt, B. Scurlock, D. Wang, J. Yelton

\section{Florida International University, Miami, USA}

C. Ceron, V. Gaultney, L. Kramer, L.M. Lebolo, S. Linn, P. Markowitz, G. Martinez, J.L. Rodriguez

\section{Florida State University, Tallahassee, USA}

T. Adams, A. Askew, H. Baer, M. Bertoldi, J. Chen, W.G.D. Dharmaratna, S.V. Gleyzer, J. Haas, S. Hagopian, V. Hagopian, M. Jenkins, K.F. Johnson, E. Prettner, H. Prosper, S. Sekmen

\section{Florida Institute of Technology, Melbourne, USA}

M.M. Baarmand, S. Guragain, M. Hohlmann, H. Kalakhety, H. Mermerkaya, R. Ralich, I. Vodopiyanov

\section{University of Illinois at Chicago (UIC), Chicago, USA}

B. Abelev, M.R. Adams, I.M. Anghel, L. Apanasevich, V.E. Bazterra, R.R. Betts, J. Callner, M.A. Castro, R. Cavanaugh, C. Dragoiu, E.J. Garcia-Solis, C.E. Gerber, D.J. Hofman, S. Khalatian, C. Mironov, E. Shabalina, A. Smoron, N. Varelas 
The University of Iowa, Iowa City, USA

U. Akgun, E.A. Albayrak, A.S. Ayan, B. Bilki, R. Briggs, K. Cankocak ${ }^{36}$, K. Chung, W. Clarida, P. Debbins, F. Duru, F.D. Ingram, C.K. Lae, E. McCliment, J.-P. Merlo, A. Mestvirishvili, M.J. Miller, A. Moeller, J. Nachtman, C.R. Newsom, E. Norbeck, J. Olson, Y. Onel, F. Ozok, J. Parsons, I. Schmidt, S. Sen, J. Wetzel, T. Yetkin, K. Yi

Johns Hopkins University, Baltimore, USA

B.A. Barnett, B. Blumenfeld, A. Bonato, C.Y. Chien, D. Fehling, G. Giurgiu, A.V. Gritsan, Z.J. Guo, P. Maksimovic, S. Rappoccio, M. Swartz, N.V. Tran, Y. Zhang

The University of Kansas, Lawrence, USA

P. Baringer, A. Bean, O. Grachov, M. Murray, V. Radicci, S. Sanders, J.S. Wood, V. Zhukova

Kansas State University, Manhattan, USA

D. Bandurin, T. Bolton, K. Kaadze, A. Liu, Y. Maravin, D. Onoprienko, I. Svintradze, Z. Wan

Lawrence Livermore National Laboratory, Livermore, USA

J. Gronberg, J. Hollar, D. Lange, D. Wright

University of Maryland, College Park, USA

D. Baden, R. Bard, M. Boutemeur, S.C. Eno, D. Ferencek, N.J. Hadley, R.G. Kellogg, M. Kirn, S. Kunori, K. Rossato, P. Rumerio, F. Santanastasio, A. Skuja, J. Temple, M.B. Tonjes, S.C. Tonwar, T. Toole, E. Twedt

Massachusetts Institute of Technology, Cambridge, USA

B. Alver, G. Bauer, J. Bendavid, W. Busza, E. Butz, I.A. Cali, M. Chan, D. D'Enterria, P. Everaerts, G. Gomez Ceballos, K.A. Hahn, P. Harris, S. Jaditz, Y. Kim, M. Klute, Y.-J. Lee, W. Li, C. Loizides, T. Ma, M. Miller, S. Nahn, C. Paus, C. Roland, G. Roland, M. Rudolph, G. Stephans, K. Sumorok, K. Sung, S. Vaurynovich, E.A. Wenger, B. Wyslouch, S. Xie, Y. Yilmaz, A.S. Yoon

University of Minnesota, Minneapolis, USA

D. Bailleux, S.I. Cooper, P. Cushman, B. Dahmes, A. De Benedetti, A. Dolgopolov, P.R. Dudero, R. Egeland, G. Franzoni, J. Haupt, A. Inyakin ${ }^{37}$, K. Klapoetke, Y. Kubota, J. Mans, N. Mirman, D. Petyt, V. Rekovic, R. Rusack, M. Schroeder, A. Singovsky, J. Zhang

University of Mississippi, University, USA

L.M. Cremaldi, R. Godang, R. Kroeger, L. Perera, R. Rahmat, D.A. Sanders, P. Sonnek, D. Summers

University of Nebraska-Lincoln, Lincoln, USA

K. Bloom, B. Bockelman, S. Bose, J. Butt, D.R. Claes, A. Dominguez, M. Eads, J. Keller, T. Kelly, I. Kravchenko, J. Lazo-Flores, C. Lundstedt, H. Malbouisson, S. Malik, G.R. Snow

State University of New York at Buffalo, Buffalo, USA

U. Baur, I. Iashvili, A. Kharchilava, A. Kumar, K. Smith, M. Strang

Northeastern University, Boston, USA

G. Alverson, E. Barberis, O. Boeriu, G. Eulisse, G. Govi, T. McCauley, Y. Musienko ${ }^{38}$, S. Muzaffar, I. Osborne, T. Paul, S. Reucroft, J. Swain, L. Taylor, L. Tuura

Northwestern University, Evanston, USA

A. Anastassov, B. Gobbi, A. Kubik, R.A. Ofierzynski, A. Pozdnyakov, M. Schmitt, S. Stoynev, M. Velasco, S. Won

University of Notre Dame, Notre Dame, USA

L. Antonelli, D. Berry, M. Hildreth, C. Jessop, D.J. Karmgard, T. Kolberg, K. Lannon, S. Lynch, 
N. Marinelli, D.M. Morse, R. Ruchti, J. Slaunwhite, J. Warchol, M. Wayne

The Ohio State University, Columbus, USA

B. Bylsma, L.S. Durkin, J. Gilmore ${ }^{39}$, J. Gu, P. Killewald, T.Y. Ling, G. Williams

Princeton University, Princeton, USA

N. Adam, E. Berry, P. Elmer, A. Garmash, D. Gerbaudo, V. Halyo, A. Hunt, J. Jones, E. Laird, D. Marlow, T. Medvedeva, M. Mooney, J. Olsen, P. Piroué, D. Stickland, C. Tully, J.S. Werner, T. Wildish, Z. Xie, A. Zuranski

University of Puerto Rico, Mayaguez, USA

J.G. Acosta, M. Bonnett Del Alamo, X.T. Huang, A. Lopez, H. Mendez, S. Oliveros, J.E. Ramirez Vargas, N. Santacruz, A. Zatzerklyany

Purdue University, West Lafayette, USA

E. Alagoz, E. Antillon, V.E. Barnes, G. Bolla, D. Bortoletto, A. Everett, A.F. Garfinkel, Z. Gecse, L. Gutay, N. Ippolito, M. Jones, O. Koybasi, A.T. Laasanen, N. Leonardo, C. Liu, V. Maroussov, P. Merkel, D.H. Miller, N. Neumeister, A. Sedov, I. Shipsey, H.D. Yoo, Y. Zheng

Purdue University Calumet, Hammond, USA

P. Jindal, N. Parashar

Rice University, Houston, USA

V. Cuplov, K.M. Ecklund, F.J.M. Geurts, J.H. Liu, D. Maronde, M. Matveev, B.P. Padley, R. Redjimi, J. Roberts, L. Sabbatini, A. Tumanov

University of Rochester, Rochester, USA

B. Betchart, A. Bodek, H. Budd, Y.S. Chung, P. de Barbaro, R. Demina, H. Flacher, Y. Gotra, A. Harel, S. Korjenevski, D.C. Miner, D. Orbaker, G. Petrillo, D. Vishnevskiy, M. Zielinski

The Rockefeller University, New York, USA

A. Bhatti, L. Demortier, K. Goulianos, K. Hatakeyama, G. Lungu, C. Mesropian, M. Yan

Rutgers, the State University of New Jersey, Piscataway, USA

O. Atramentov, E. Bartz, Y. Gershtein, E. Halkiadakis, D. Hits, A. Lath, K. Rose, S. Schnetzer, S. Somalwar, R. Stone, S. Thomas, T.L. Watts

University of Tennessee, Knoxville, USA

G. Cerizza, M. Hollingsworth, S. Spanier, Z.C. Yang, A. York

Texas A\&M University, College Station, USA

J. Asaadi, A. Aurisano, R. Eusebi, A. Golyash, A. Gurrola, T. Kamon, C.N. Nguyen, J. Pivarski, A. Safonov, S. Sengupta, D. Toback, M. Weinberger

Texas Tech University, Lubbock, USA

N. Akchurin, L. Berntzon, K. Gumus, C. Jeong, H. Kim, S.W. Lee, S. Popescu, Y. Roh, A. Sill, I. Volobouev, E. Washington, R. Wigmans, E. Yazgan

Vanderbilt University, Nashville, USA

D. Engh, C. Florez, W. Johns, S. Pathak, P. Sheldon

University of Virginia, Charlottesville, USA

D. Andelin, M.W. Arenton, M. Balazs, S. Boutle, M. Buehler, S. Conetti, B. Cox, R. Hirosky, A. Ledovskoy, C. Neu, D. Phillips II, M. Ronquest, R. Yohay

Wayne State University, Detroit, USA

S. Gollapinni, K. Gunthoti, R. Harr, P.E. Karchin, M. Mattson, A. Sakharov 


\section{University of Wisconsin, Madison, USA}

M. Anderson, M. Bachtis, J.N. Bellinger, D. Carlsmith, I. Crotty ${ }^{1}$, S. Dasu, S. Dutta, J. Efron, F. Feyzi, K. Flood, L. Gray, K.S. Grogg, M. Grothe, R. Hall-Wilton ${ }^{1}$, M. Jaworski, P. Klabbers, J. Klukas, A. Lanaro, C. Lazaridis, J. Leonard, R. Loveless, M. Magrans de Abril, A. Mohapatra, G. Ott, G. Polese, D. Reeder, A. Savin, W.H. Smith, A. Sourkov ${ }^{40}$, J. Swanson, M. Weinberg, D. Wenman, M. Wensveen, A. White

$\dagger$ : Deceased

1: Also at CERN, European Organization for Nuclear Research, Geneva, Switzerland

2: Also at Universidade Federal do ABC, Santo Andre, Brazil

3: Also at Soltan Institute for Nuclear Studies, Warsaw, Poland

4: Also at Université de Haute-Alsace, Mulhouse, France

5: Also at Centre de Calcul de l'Institut National de Physique Nucleaire et de Physique des Particules (IN2P3), Villeurbanne, France

6: Also at Moscow State University, Moscow, Russia

7: Also at Institute of Nuclear Research ATOMKI, Debrecen, Hungary

8: Also at University of California, San Diego, La Jolla, USA

9: Also at Tata Institute of Fundamental Research - HECR, Mumbai, India

10: Also at University of Visva-Bharati, Santiniketan, India

11: Also at Facolta' Ingegneria Universita' di Roma "La Sapienza", Roma, Italy

12: Also at Università della Basilicata, Potenza, Italy

13: Also at Laboratori Nazionali di Legnaro dell' INFN, Legnaro, Italy

14: Also at Università di Trento, Trento, Italy

15: Also at ENEA - Casaccia Research Center, S. Maria di Galeria, Italy

16: Also at Warsaw University of Technology, Institute of Electronic Systems, Warsaw, Poland

17: Also at California Institute of Technology, Pasadena, USA

18: Also at Faculty of Physics of University of Belgrade, Belgrade, Serbia

19: Also at Laboratoire Leprince-Ringuet, Ecole Polytechnique, IN2P3-CNRS, Palaiseau, France 20: Also at Alstom Contracting, Geneve, Switzerland

21: Also at Scuola Normale e Sezione dell' INFN, Pisa, Italy

22: Also at University of Athens, Athens, Greece

23: Also at The University of Kansas, Lawrence, USA

24: Also at Institute for Theoretical and Experimental Physics, Moscow, Russia

25: Also at Paul Scherrer Institut, Villigen, Switzerland

26: Also at Vinca Institute of Nuclear Sciences, Belgrade, Serbia

27: Also at University of Wisconsin, Madison, USA

28: Also at Mersin University, Mersin, Turkey

29: Also at Izmir Institute of Technology, Izmir, Turkey

30: Also at Kafkas University, Kars, Turkey

31: Also at Suleyman Demirel University, Isparta, Turkey

32: Also at Ege University, Izmir, Turkey

33: Also at Rutherford Appleton Laboratory, Didcot, United Kingdom

34: Also at INFN Sezione di Perugia; Universita di Perugia, Perugia, Italy

35: Also at KFKI Research Institute for Particle and Nuclear Physics, Budapest, Hungary

36: Also at Istanbul Technical University, Istanbul, Turkey

37: Also at University of Minnesota, Minneapolis, USA

38: Also at Institute for Nuclear Research, Moscow, Russia

39: Also at Texas A\&M University, College Station, USA

40: Also at State Research Center of Russian Federation, Institute for High Energy Physics, Protvino, Russia 
